\title{
Hydrochemical Classification of Groundwater in West Assiut Combined Cycle Power's Area, Assiut, Egypt
}

\author{
Abdel-Aal M. Gaber ${ }^{1,2}$, Adel M. Kamal-El-Dean²,Hassanien H. \\ Manaa $^{3}$, Ramy M. Dissoky ${ }^{4}$ \\ ${ }^{1}$ Faculty of Sugar and Integrated Industries Technology, Assiut \\ University, Assiut, Egypt \\ ${ }^{2}$ Chemistry Department, Faculty of Science, Assiut University, Assiut, \\ Egypt \\ ${ }^{3}$ General Manger of Chemistry department, WACCPP, Egyp. \\ ${ }^{4}$ Shift Supervisor Chemist, WACCPP, Egypt
}

\begin{abstract}
Groundwater is the main source for water used in West Assiut Electric Power Plant (WACCPP), so here we will study the hydrochemical class and water type for WACCPP area's groundwater, with respect to the physiochemical, chemical elements and chemically related properties. Hydrochemical formulae's as Kurolov formula, hypothetical salts, hydrochemical indices and water type identification programs as Piper, Durov, Schoeller, Gibb, and Chadha will be applied for deciding the water class and type.
\end{abstract}

Keywords: Groundwater, WACCPP, hydrochemical parameters, Hypothetical salts, Hydrochemical indicators, Hydrochemical faces.

\section{Introduction}

Water is being used for electric power generation and different purposes nowadays from traditional sources as Nile River and, nontraditional water sources such as seawater, excessively hard or brackish groundwater, poorer quality surface waters, and wastewater [1]. These sources commonly require treatment with high quality technologies before use. In West Assiut Combined Cycle Power Plant (WACCPP) groundwater is used as the main source for demineralized water production, for this purpose, 37 wells were drilled there. In this paper, we will examine the type and hydrochemical class of the area's groundwater. The groundwater is considered the source of water for domestic, agricultural and 
industrial uses in many Arab countries [2]. This demand has faced by the groundwater quality that affected by the following processes: Chemical reactions with geological materials, geochemical reactions, biodegradation, Dissolution [2] and exsolution of gases [3] and anthropogenic pollution[4]. The water quality is in equal importance to its quantity, so it is necessary to analyze groundwater chemical, physiochemical and hydrochemical properties, any deviation from the standard value for one or more of a water quality's parameters, water is considered polluted. Also, determining water type and quality depend on the hydrochemical parameters for the groundwater as hydrochemical formula (Kurolov formula) and water type, hypothetical Salts, hydrochemical indicators and index of base exchange.

On the other hand, methods are used for hydrochemical classification of groundwater are Piper, Piper and Langguth, Durov, Schoeller, Gibband Chadha, those are constructed depending on the main cations and anions concentrations by unit equivalent weight of ion in mills equivalent per liter(epm) and epm \%.

\section{Experimental}

\subsection{Materials and Methods}

\subsubsection{Study Area}

Assiut is the largest town in Upper Egypt and lies about 234 miles south of Cairo. The city of Assiut is located at $27^{\circ} 11^{\prime} 00^{\circ} \mathrm{N}$, $31^{\circ} 10^{\circ} 00^{\circ} \mathrm{E}$ and spread across $26,000 \mathrm{~km} 2$. WACCPP is located at the northern west of Assiut city in Assiut governorate in Upper Egypt, and allocated from Assiut city by $25 \mathrm{~km}$ and at about $5 \mathrm{~km}$ from Bany Ghalleb and about $3.43 \mathrm{~km}$ from the nearest village Jhdum, Located between the Petroleum Company in the north and cement company in the south, it is on a 33.6 acres area. The wells were lied in the south, east, and west sides of the area, the water depth is about $80 \mathrm{~m}$ and the overall wells deep is $200 \mathrm{~m}$. In WACCPP, power plants use the demineralized water for : Steam generation, cooling, sealing, de-NOx, washing and so on. West Assiut power plant specifications, Table (1). 
Table (1): Description of WACCPP's Constituents

\begin{tabular}{|c|c|}
\hline Item & Description \\
\hline Simple cycle & $\begin{array}{l}\text { Two Modules of gas turbines, contains } 8 \text { gas turbine units, power } \\
\text { capacity. }\end{array}$ \\
\hline Power capacity & $125 \mathrm{MW} / \mathrm{hr}$ for each gas turbine \\
\hline Exhaust gases temp. & $590^{\circ} \mathrm{C}$ \\
\hline Water uses & $\begin{array}{l}176 \text { ton/hr injection waters for } \mathrm{d}-\mathrm{NOx} \text {, Makeup water for closed } \\
\text { cooling system, Washing water for the turbine and compressor } \\
\text { offline washing }\end{array}$ \\
\hline \multicolumn{2}{|c|}{$\begin{array}{l}\text { Description the constituents of combined cycle stage's } \\
\end{array}$} \\
\hline Combined cycle & Two Modules of HRSGs, one on each module of simple cycle \\
\hline Power capacity & $250 \mathrm{MW} / \mathrm{hr}$ for each module \\
\hline Exhaust gases temp. & $150{ }^{\circ} \mathrm{C}$ \\
\hline Water uses & $\begin{array}{l}\text { Makeup water for closed thermal cycle, Makeup water for closed } \\
\text { cooling system, Washing water for the HRSGS offline washing }\end{array}$ \\
\hline Steam ton $/ \mathrm{hr}$ & 840 ton/hr for each combined \\
\hline Steam pressure bar & 116 \\
\hline
\end{tabular}

\subsubsection{Sampling and analytical procedures (Measure of Parameters)}

Water samples from the thirty seven wells and raw water(mixture tank) in WACCPP (study area) were withdrawn and sample's bottles were prepared according the ASTM standards and the water samples were taken under almost careful conditions after the water was pumped out for about 10 minutes to remove the stagnant water. All samples were preserved in refrigerator before chemical analysis. The samples were analyzed for determining its content of : pH, EC, TDS. Also trace elements were analyzed as important cations like Calcium $\left(\mathrm{Ca}^{2+}\right)$, magnesium $\left(\mathrm{Mg}^{2+}\right)$, sodium $\left(\mathrm{Na}^{+}\right)$, potassium $\left(\mathrm{K}^{+}\right)$and anions like bicarbonate $\left(\mathrm{HCO}^{-3}\right)$, Chloride $\left(\mathrm{Cl}^{-}\right)$, Nitrate $\left(\mathrm{NO}^{-3}\right)$, Carbonate $\left(\mathrm{CO}^{-3}\right)$ and Sulfate $\left(\mathrm{SO}_{4}^{-2}\right)$. the measurements were conducted according to the standard specification using; HANA (HI9811-5) instrument volumetric method[5], flame photometer[6], titration[7], ultraviolet spectra (U.V), atomic absorption [8].All concentrations were expressed in milligrams per liter (mg/L), except for $\mathrm{pH}$ and EC. Ionic-balanceerror was checked for accuracy of each groundwater sample, which is generally within acceptable limit of $\pm 5 \%$. 


\subsubsection{Determination of groundwater type was done using : \\ 1- Hydrochemical formula (kurolov formula)}

It depends on the ratio of cation and anions, which have ratio of availability more than $15 \%$ as in Eq.(1) [9]:

TDS $m g / L$

$=\frac{\text { Anions epm } \% \text { decreasing order }}{\text { cations epm } \% \text { decreasing order }} p H$

\section{2- Hypothetical salts}

Hypothetically, the ions of strong acids $\mathrm{Cl}^{-}$and $\mathrm{SO}_{4}{ }^{2-}$, form a chemical combination with alkalis, $\mathrm{Na}^{+}$and $\mathrm{K}^{+}$and the rest of acid radicals combine with alkaline earths $\mathrm{Ca}^{2+}$ and $\mathrm{Mg}^{2+}[10]$. If the cations of alkali and alkaline earths all in epm \% are surplus in groundwater [11], they will combine with anions of the weak acids $\mathrm{CO}_{3}{ }^{2-}$ and $\mathrm{HCO}_{3}{ }^{-}[10,12]$.

\section{3- Hydrochemical Indicators}

They were used to determine the origin of the groundwater; meteoric or marine water and helped in discovering the previous hydrochemical processes affecting water quality [9]. These indicators are a ratio between the different major ions. $\mathrm{Cl}^{-}$ion is considered a well defining mean in determining the origin of water, (where the ratio of main elements to $\mathrm{Cl}^{-}$gives geochemical behavior for the main elements) [13].

\section{4- Index of Base Exchange}

The exchange between alkali metals cations and alkaline earth metals cations in the water and its host environment during residence or travel periods could be determined by the chloroalkaline indices (CAI) [14, 15]

\subsubsection{Hydrochemical classification of groundwater}

Using Aquachem v3.7 software for better understanding of hydrochemistry, water quality and its evaluation by comparing the water types and to interpret variation in hydrochemical processes. Piper [16], Durov [17], Schoeller [18], Gibb [19] and Chadha [20] diagrams were constructed depending on the main cations and anions concentrations by unit equivalent weight of ion in mille equivalent per liter (epm) and epm \% [20]. 


\subsubsection{Hydrochemical Faces}

The hydrochemical faces has been studied based on Chebotarev sequence[4, 21]. In Chebotarev sequence, he proposed a geochemical classification of water based on anion occurrence and prevalence as they are regarded as independent ingredients, the chemical quality of groundwater is classified into three major groups according to major anions and TDS, respectively [21].

\section{Results and Discussion}

\subsection{Physical Specifications Results}

Wells and Raw water sample's physical analysis results as odor, color, taste, $\mathrm{pH}$, TDS and EC were analyzed and determined as in Table (2)

Table (2): Analysis of physical parameters of water samples

\begin{tabular}{|l|l|l|l|l|l|l|l|l|l|l|l|l|l|l|l|l|l|l|l|l|}
\hline Item & $1 \mathrm{~A}$ & $1 \mathrm{~B}$ & $2 \mathrm{~A}$ & $2 \mathrm{~B}$ & $3 \mathrm{~A}$ & $3 \mathrm{~B}$ & $4 \mathrm{~A}$ & $4 \mathrm{~B}$ & $5 \mathrm{~A}$ & $5 \mathrm{~B}$ & $6 \mathrm{~A}$ & $6 \mathrm{~B}$ & $7 \mathrm{~A}$ & $7 \mathrm{~B}$ & $8 \mathrm{~A}$ & $8 \mathrm{~B}$ & $9 \mathrm{~A}$ & $9 \mathrm{~B}$ & $10 \mathrm{~A}$ & $10 \mathrm{~B}$ \\
\hline EC & 2540 & 4980 & 2700 & 3300 & 1924 & 5130 & 1034 & 1446 & 2060 & 2260 & 1736 & 2580 & 1323 & 1393 & 3120 & 1557 & 2650 & 1469 & 1730 & 2980 \\
\hline pH & 8.3 & 7.75 & 8.39 & 7.71 & 8.3 & 7.55 & 8.4 & 8.34 & 8.37 & 7.59 & 8.4 & 7.61 & 7.85 & 8.02 & 7.34 & 7.88 & 7.67 & 7.75 & 8.39 & 7.82 \\
\hline TDS & 1625 & 3187 & 1720 & 2112 & 1231 & 3283 & 663 & 925 & 1319 & 1438 & 1114 & 1651 & 846 & 891 & 1997 & 996 & 1696 & 940 & 1104 & 1910 \\
\hline
\end{tabular}

\section{Table (2); continued}

\begin{tabular}{|c|c|c|c|c|c|c|c|c|c|c|c|c|c|c|c|c|c|c|}
\hline Item & $11 \mathrm{~A}$ & $11 \mathrm{~B}$ & $12 \mathrm{~A}$ & $12 \mathrm{~B}$ & $13 \mathrm{~A}$ & $13 \mathrm{~B}$ & $14 \mathrm{~A}$ & $14 \mathrm{~B}$ & $15 \mathrm{~A}$ & $15 \mathrm{~B}$ & $16 \mathrm{~A}$ & $17 \mathrm{~A}$ & $18 \mathrm{~A}$ & $19 \mathrm{~A}$ & $19 \mathrm{~B}$ & $20 \mathrm{~A}$ & $20 \mathrm{~B}$ & Raw \\
\hline EC & 2120 & 2610 & 1690 & 2940 & 4000 & 4165 & 5061 & 4080 & 1473 & 1847 & 2190 & 1283 & 1156 & 1170 & 1914 & 1004 & 1509 & 4680 \\
\hline pH & 7.89 & 7.05 & 7.81 & 7.74 & 7.65 & 7.9 & 7.7 & 7.8 & 8.1 & 7.87 & 7.45 & 7.94 & 8.38 & 8.46 & 7.89 & 8.33 & 7.6 & 7.38 \\
\hline TDS & 1356 & 1669 & 1081 & 1881 & 2400 & 2707 & 3280 & 2652 & 943 & 1182 & 1404 & 821 & 740 & 749 & 1224 & 643 & 966 & 2858 \\
\hline
\end{tabular}

From Table (2), it can be noted that PH values vary between 7.05 and 8.46, reflect neutral to slightly alkaline water, electric conductivity varies between 1004 and $5130 \mu \mathrm{s} / \mathrm{cm}$, so wells and raw water are excessively mineralized water, TDS content ranging from 643 to $3283 \mathrm{ppm}$, so wells and Raw water are fresh to slightly brackish water.

\subsection{Chemical Specifications Results}

Water samples were analyzed for major cations, major anions, and heavy elements, as shown in Table (3). 


\begin{tabular}{|c|c|c|c|c|c|c|c|c|c|c|c|c|c|c|c|c|c|c|c|}
\hline & & & & & & & & & & & & & & & & & & & \\
\hline Item & $\mathbf{1 A}$ & $1 B$ & $2 A$ & 2B & $\mathbf{3 A}$ & 3B & $4 A$ & 4B & $5 \mathbf{A}$ & 5B & $6 A$ & $6 B$ & 7A & 7B & $\mathbf{8 A}$ & 8B & 9A & 9B & 10A \\
\hline Cl & 580 & 1320 & 748 & 825 & 540 & 1420 & 192 & 305 & 508 & 505 & 457 & 573 & 279 & 260 & 682 & 325 & 587 & 288 & 399 \\
\hline $\mathrm{CO}_{3}$ & 16 & $\mathbf{0}$ & $\mathbf{0}$ & $\mathbf{0}$ & 26 & $\mathbf{0}$ & 28 & 10 & 13 & 0 & 26 & $\mathbf{0}$ & $\mathbf{0}$ & $\mathbf{0}$ & $\mathbf{0}$ & $\mathbf{0}$ & $\mathbf{0}$ & $\mathbf{0}$ & 12 \\
\hline $\mathrm{HCO}_{3}$ & 268 & 180 & 150 & 278 & 217 & 241 & 250 & 225 & 252 & 225 & 322 & 214 & 325 & 285 & 229 & 225 & 175 & 310 & 320 \\
\hline $\mathrm{NO}_{3}$ & 5.9 & 7.4 & 0.2 & 8.8 & 11.2 & 0.2 & 0 & 3.7 & 5.7 & 5.6 & 8.2 & 13.5 & 0.7 & 0.2 & 65.5 & 12.3 & 33.6 & 6.2 & 10 \\
\hline $\mathrm{SO}_{4}$ & 185.6 & 285 & 160 & 80 & 38.6 & 163 & 20.3 & 25 & 116 & 82 & 60.6 & 165 & 11.8 & 22 & 300 & 32 & 130 & 28.3 & 60.3 \\
\hline $\mathbf{C a}$ & 75.7 & 183 & 71.5 & 74.7 & 47.2 & 171 & 17 & 20 & 65.2 & 60 & 33.5 & 77 & 14.4 & 24.6 & 154 & 25.7 & 66 & 31 & 53.3 \\
\hline $\mathbf{K}$ & 21.5 & 5 & 3 & 5 & 4 & 8 & 3 & 4 & 3.08 & 6 & 5.5 & 11 & 3 & 4 & 3 & 3 & 5 & 7 & 2.44 \\
\hline $\mathbf{M g}$ & 25.7 & 15 & 24.2 & 15 & 22.6 & 56.9 & 13.2 & 8 & 15.9 & 15 & 9.81 & 22 & 9.72 & 14.2 & 43.7 & 8 & 21 & 10 & 13.8 \\
\hline $\mathbf{N a}$ & 424 & 885 & 500 & 620 & 370 & 800 & 200 & 280 & 380 & 385 & 282 & 435 & 270 & 250 & 450 & 292 & 534 & 285 & 327 \\
\hline
\end{tabular}

Table (3); continued

\begin{tabular}{|c|c|c|c|c|c|c|c|c|c|c|c|c|c|c|c|c|c|c|c|}
\hline Item & 10B & 11A & 11B & $12 \mathrm{~A}$ & 12B & 13A & 13B & 14A & $14 B$ & $15 A$ & 15B & $16 A$ & 17A & 18A & 19A & 19B & $20 \mathrm{~A}$ & $20 B$ & Raw \\
\hline Cl & 774 & 510 & 661 & 380 & 752 & 1134 & 1250 & 1449 & 1153 & 320 & 340 & 534 & 250 & 253 & 231 & 425 & 154 & 338 & \\
\hline $\mathrm{CO}_{3}$ & $\mathbf{0}$ & $\mathbf{0}$ & $\mathbf{0}$ & $\mathbf{0}$ & $\mathbf{0}$ & 4 & 4 & 4 & 4 & $\mathbf{0}$ & $\mathbf{0}$ & $\mathbf{0}$ & $\mathbf{0}$ & 24 & & & 13 & $\mathbf{0}$ & \\
\hline $\mathrm{HCO}_{3}$ & 259 & 20 & & 240 & 243 & & & & 150 & 200 & 326 & & & 200 & & & & & \\
\hline $\mathrm{NO}_{3}$ & 6.2 & 135 & 47 & 7.1 & 3.9 & 60 & 53.6 & 6 & 31.2 & 20.2 & 0.2 & 15.3 & 0.29 & $\mathbf{0}$ & 2 & 0.2 & 0.2 & 0.6 & 1.4 \\
\hline $\mathrm{SO}_{4}$ & 110 & 100 & 110 & 93.7 & 104 & 235 & 185 & 327 & 187 & 59.8 & 44 & 138 & 34 & 31.9 & 32.8 & 62.3 & 57 & 41 & 250 \\
\hline $\mathbf{C a}$ & 80.7 & 76.4 & 54.2 & 62.2 & 48.2 & 160 & & 183 & 136 & 29.7 & 52.1 & 110 & 20.4 & 29.3 & & 35 & & 33 & 200 \\
\hline & 5 & 5 & 7 & 2.65 & 6 & 4.6 & 5.9 & 5 & 5.7 & 4 & 4 & 4 & 2 & 2 & 1.17 & 3 & 1.26 & 3 & 40 \\
\hline & 15 & 16.2 & 11 & 26.9 & 33.8 & 61 & 01 & 13 & 49 & 3.49 & 12.2 & 40 & 10.2 & 16.3 & 16.8 & 14 & 14.7 & 9 & 50 \\
\hline $\mathbf{N a}$ & 575 & 350 & 485 & 254 & 500 & 611 & 610 & 721 & 616 & 280 & 280 & 310 & 240 & 204 & 200 & 370 & 175 & 290 & 595 \\
\hline
\end{tabular}




\subsubsection{Data Processing}

The results in ppm, epm (meq/l) and in epm\% is as shown in Table (4). Also, it was subjected to correctness and instrumentation validity and hydrochemical classification.

Table 4); (major ions+ $\mathrm{CO}_{3}$ and $\mathrm{NO}_{3}$ ) as epm and epm\%

\begin{tabular}{|c|c|c|c|c|c|c|c|c|c|c|c|c|c|c|c|c|c|c|c|}
\hline Item & $1 \mathrm{~A}$ & 1B & $2 \mathrm{~A}$ & $2 \mathrm{~B}$ & $3 \mathrm{~A}$ & 3B & $4 \mathrm{~A}$ & $4 \mathrm{~B}$ & $5 \mathrm{~A}$ & $5 B$ & $6 \mathrm{~A}$ & $6 \mathrm{~B}$ & $7 \mathrm{~A}$ & $7 \mathrm{~B}$ & $8 \mathrm{~A}$ & $8 \mathrm{~B}$ & $9 \mathrm{~A}$ & 9B & $10 \mathrm{~A}$ \\
\hline CO3 epm & 0.53 & 0 & 0 & 0 & 0.87 & 0 & 0.93 & 0.33 & 0.43 & 0 & 0.87 & 0 & 0 & 0 & 0 & 0 & 0 & 0 & 0.4 \\
\hline C03 ерm\% & 2.11 & 0 & 0 & 0 & 4.3 & 0 & 8.6 & 2.51 & 2.01 & 0 & 4.3 & 0 & 0 & 0 & 0 & 0 & 0 & 0 & 2.2 \\
\hline Clepm & 16.3 & 37.2 & 21 & 23.2 & 15.2 & 40 & 5.4 & 8.6 & 14.3 & 14.2 & 12.9 & 16.1 & 7.86 & 7.32 & 19.2 & 9.15 & 16.5 & 8.1 & 11.2 \\
\hline Clepm $\%$ & 64.7 & 80.5 & 78.4 & 78.5 & 73.7 & 84.5 & 49.8 & 65.2 & 66.9 & 72.1 & 63.1 & 69.2 & 58.4 & 58.8 & 63.5 & 59 & 72.9 & 58.4 & 61.3 \\
\hline HCO3 epm & 4.39 & 2.95 & 2.46 & 4.56 & 3.56 & 3.95 & 4.1 & 3.69 & 4.13 & 3.69 & 5.28 & 3.51 & 5.33 & 4.67 & 3.75 & 3.69 & 2.87 & 5.08 & 5.25 \\
\hline HCO3 $\mathrm{pm} \%$ & 17.4 & 6.4 & 9.2 & 15.4 & 17.2 & 8.34 & 37.8 & 27.9 & 19.3 & 18.7 & 25.8 & 15.1 & 39.6 & 37.5 & 12.4 & 23.8 & 12.7 & 36.6 & 28.7 \\
\hline NO3 epm & 0.09 & 0.12 & 0.002 & 0.14 & 0.18 & 0.003 & 0 & 0.06 & 0.09 & 0.09 & 0.13 & 0.217 & 0.011 & 0.003 & 1.056 & 2 & 0.54 & 0.1 & 0.16 \\
\hline NO3 em\% & 0.36 & 0.25 & 0.01 & 0.47 & 0.87 & 0.01 & 0 & 0.45 & 0.42 & 0.46 & 0.64 & 0.93 & \begin{tabular}{|l|l|}
0.08 \\
\end{tabular} & 0.02 & 3.49 & 12.9 & 2.4 & 0.72 & 0.87 \\
\hline SO4 epm & 3.87 & 5.94 & 3.33 & 1.67 & 0.8 & 3.4 & 0.42 & 0.52 & 2.42 & 1.71 & 1.26 & 3.44 & 0.25 & 0.46 & 6.25 & 0.67 & 2.71 & 0.59 & 1.26 \\
\hline S04 epm\% & 15.4 & 12.8 & 12.4 & 5.6 & 3.88 & 7.18 & 3.87 & 3.94 & 11.3 & 8.68 & 6.16 & 14.8 & 1.86 & 3.69 & 20.6 & 4.32 & 12 & 4.25 & 6.89 \\
\hline Sum epm & 25.2 & 46.2 & 26.8 & 29.6 & 20.6 & 47.4 & 10.8 & 13.2 & 21.4 & 19.7 & 20.4 & 23.3 & 13.5 & 12.5 & 30.3 & 15.5 & 22.6 & 18.2 & 13.8 \\
\hline Caepm & 3.8 & 9.15 & 3.6 & 3.73 & 2.36 & 8.55 & 0.85 & 1 & 3.26 & 3 & 1.7 & 3.85 & 0.72 & 1.23 & 7.7 & 1.3 & 3.3 & 1.55 & 2.7 \\
\hline Caepm\% & 15.3 & 18.7 & 13.1 & 11.6 & 11.5 & 17.7 & 7.93 & 7.16 & 15.4 & 14.2 & 11.4 & 15.5 & 5.41 & 9.18 & 24.8 & 8.82 & 11.6 & 10.4 & 14.9 \\
\hline Kepm & 0.55 & 0.13 & 0.08 & 0.13 & 0.1 & 0.2 & 0.08 & 0.1 & 0.08 & 0.15 & 0.14 & 0.28 & 0.08 & 0.1 & 0.08 & 0.08 & 0.13 & 0.18 & 0.06 \\
\hline Kepm\% $\%$ & 2.21 & 0.27 & 0.29 & 0.41 & 0.49 & 0.41 & 0.75 & 0.71 & 0.38 & 0.71 & 0.93 & 1.13 & 0.6 & 0.75 & 0.26 & 0.543 & 0.46 & 1.2 & 0.33 \\
\hline Mgepm & 2.1 & 1.2 & 1.99 & 1.23 & 1.86 & 4.68 & 1.08 & 0.66 & 1.31 & 1.23 & 0.81 & 1.81 & 0.8 & 1.17 & 3.6 & 0.66 & 1.72 & 0.82 & 1.13 \\
\hline Mgepm $\%$ & 8.45 & 2.45 & 7.27 & 3.83 & 9.11 & 9.7 & 10.1 & 4.73 & 6.2 & 5.83 & 5.42 & 7.29 & 6.01 & 8.73 & 11.62 & 4.48 & 6.07 & 5.48 & 6.24 \\
\hline Naepm & 18.4 & 38.5 & 21.7 & 27 & 16.1 & 34.8 & 8.7 & 12.2 & 16.5 & 16.7 & 12.3 & 18.9 & 11.7 & 10.9 & 19.6 & 12.7 & 23.2 & 12.4 & 14.2 \\
\hline $\mathrm{Na} \mathrm{epm \%} \%$ & 74 & 78.6 & 79.3 & 84.1 & 78.8 & 72.1 & 81.2 & 87.4 & 78 & 79.2 & 82.3 & 76.1 & 87.97 & 81.34 & 63.27 & 86.16 & 81.8 & 82.9 & 78.5 \\
\hline Sum epm & 24.85 & 48.98 & 27.37 & 32.09 & 20.42 & 48.23 & & 13.96 & 21.15 & 21.08 & 14.95 & 24.84 & 13.3 & 13.4 & 30.98 & 14.74 & 28.35 & 18.09 & 14.95 \\
\hline
\end{tabular}


Table (4) continued.

\begin{tabular}{|c|c|c|c|c|c|c|c|c|c|c|c|c|c|c|c|c|c|c|c|}
\hline & $10 \mathrm{~B}$ & $11 \mathrm{~A}$ & 11B & $12 \mathrm{~A}$ & $12 \mathrm{~B}$ & $13 \mathrm{~A}$ & 13B & $14 \mathrm{~A}$ & $14 B$ & $15 \mathrm{~A}$ & 15B & $16 \mathrm{~A}$ & $17 \mathrm{~A}$ & $18 \mathrm{~A}$ & $19 \mathrm{~A}$ & 19B & $20 \mathrm{~A}$ & $20 \mathrm{~B}$ & Raw \\
\hline $\mathrm{CO}_{3} \mathrm{epm}$ & 0 & 0 & 0 & 0 & 0 & 0.13 & 0.13 & 0.01 & 0.13 & 0 & 0 & 0 & 0 & 0.8 & 0.83 & 0 & 0.43 & 0 & 0.68 \\
\hline C03ерm\% & 1 & 0 & 0 & 0 & 0 & 0.3 & 0.3 & 0.02 & 0.33 & 0 & & | & 0 & 6.74 & 7.27 & 0 & 4.2 & 0 & 1.45 \\
\hline Cl epm & 8 & 4.4 & 18.6 & 10.7 & 21.2 & 31.9 & 35.2 & 40.8 & 32.5 & 9.01 & 9.6 & 15 & 7 & 7.13 & 6.5 & 12 & 4.33 & 9.5 & 32.7 \\
\hline Cl epm\% $\%$ & 6.7 & 69.3 & 77 & 64.1 & 77.3 & 80.3 & 83 & 81.6 & 82.3 & 65 & 60.5 & 68.2 & 56.2 & 60.1 & 56.9 & 67.4 & 42.4 & 67.1 & 69.5 \\
\hline $\mathrm{HCO3} \mathrm{epm}$ & 4.25 & 4.1 & 3.2 & 3.93 & 3.98 & 1.82 & 2.38 & 1.39 & 2.46 & 3.28 & 5.34 & 3.85 & 4.75 & 3.28 & 3.4 & 4.51 & 4.26 & 3.79 & 8.27 \\
\hline HCO3 epm\% $\%$ & 15 & 19.7 & 13.2 & 23.5 & 14.5 & 4.58 & 5.61 & 2.78 & 6.23 & 23.6 & 33.6 & 17.5 & 38.1 & 27.6 & 29.79 & 25.3 & 41.7 & 26.8 & 17.6 \\
\hline N03 ерm & 0.098 & 0.21 & 0.07 & 0.11 & 0.06 & 0.96 & 0.86 & 0.96 & 0.5 & 0.33 & 0.003 & 0.25 & 0.004 & 0 & 0.014 & 0.002 & 0.003 & 0.009 & 0.16 \\
\hline N03 epm & 0.34 & 1.01 & 0.29 & 0.66 & 0.22 & 2.42 & 2.03 & 1.92 & 1.27 & 2.34 & 0.02 & 1.14 & 0.032 & 0 & 0.123 & 0.01 & 0.03 & 0.064 & 0.34 \\
\hline SO4 e & & & 2.29 & 1.95 & 2.17 & 4.9 & 3.85 & 6.81 & 3.9 & 1.25 & 0.92 & 2.88 & 0.71 & 0.66 & 0.68 & 1.3 & 1.19 & 0.85 & 5.2 \\
\hline S04 ерm\% & 8.05 & 10 & 9.48 & 11.7 & 7.9 & 12.3 & 9.07 & 13.6 & 9.9 & 9 & 5.8 & 13.1 & 5.69 & 5.56 & 5.95 & 7.3 & 11.6 & 6 & 11 \\
\hline Sum epm & 28.43 & 20.79 & 24.16 & 16.69 & 27.41 & 39.71 & 42.42 & 49.97 & 39.49 & 13.86 & 15.86 & 21.98 & 12.46 & 11.87 & 11.42 & 17.81 & 10.21 & 14.14 & 47,01 \\
\hline Caepm & 4.03 & 3.82 & 3.21 & 3.11 & 2.41 & 8 & 7.65 & 9.15 & 6.8 & 1.5 & 2.6 & 5.5 & 1.02 & 1.46 & 1.38 & 1.75 & 1.3 & 1.65 & 11.9 \\
\hline Ca ерт\% $\%$ & 13.2 & 18.6 & 12.4 & 18.9 & 8.91 & 20.2 & 19.4 & 19.6 & 18 & 10.6 & 16.3 & 24.5 & 8.28 & 12.4 & 12 & 9.17 & 12.8 & 10.9 & 27.7 \\
\hline K epm & 13 & 13 & 0.18 & 0.07 & 0.15 & 0.12 & 0.15 & 0.13 & 0.15 & 0.1 & 0.1 & 0.1 & 0.05 & 0.05 & 0.03 & 0.08 & 0.03 & 0.08 & 1.02 \\
\hline K ерm\% $\%$ & 0.428 & 0.635 & 0.69 & 0.427 & 0.55 & 0.303 & 0.38 & 0.279 & 0.397 & 0.71 & 0.629 & 0.446 & 0.406 & 0.427 & 0.261 & 0.42 & 0.296 & 0.53 & 2.37 \\
\hline Mg epm & 1.23 & 1.33 & 1.4 & 2.21 & 2.78 & 5.02 & 5.02 & 6 & 4.03 & 0.29 & 1 & 3.29 & 0.84 & 1.34 & 1.38 & 1.15 & 1.21 & 0.74 & 4.11 \\
\hline Mg epm\% $\%$ & 4.05 & 6.49 & 5.4 & 13.5 & 10.3 & 12.7 & 12.7 & 12.9 & 10.7 & 2.06 & 6.29 & 14.7 & 6.82 & 11.4 & 12.01 & 6.03 & 11.9 & 4.91 & 9.57 \\
\hline Na epm & 25 & 15.2 & 21.1 & 11 & 21.7 & 26.5 & 26.5 & 31.3 & 26.8 & 12.2 & 12.2 & 13.5 & 10.4 & 8.87 & 8.7 & 16.1 & 7.6 & 12.6 & 25.9 \\
\hline Na epm\% & 82.2 & 74.2 & 81.5 & 67.1 & 80.2 & 66.8 & 67.4 & 67.2 & 70.9 & 86.6 & 76.73 & 60.3 & 84.5 & 75.7 & 75.7 & 84.4 & 74.9 & 83.6 & 60.3 \\
\hline Sum epm & 30.39 & 20.48 & 25.89 & 16.39 & 27.04 & 39.64 & 39.32 & 46.58 & 37.78 & 14.09 & 15.9 & 22.39 & 12.31 & 11.72 & 11.49 & 19.08 & 10.14 & 15.07 & 42.93 \\
\hline
\end{tabular}




\subsection{Analyses' Correctness and Instrumentation Validity}

A few samples with ionic concentrations above 50 epm have been excluded from the analysis, the procedures for checking analyses' correctness and instruments' accuracy as ionic balance and TDS-toEC ratio were applied to water samples analyses' results.

\section{1- Ionic balance error \%:}

The anion and cation sums must balance because water is electrically neutral, the ionic balance \% (U), is equal to the absolute difference between total of cations and anions concentration on total for these concentrations in epm, and calculated using Eqs. (2,3) $[22,23]$. When $U \leq 5$, the result could be accepted, but if $5<U \leq 10$ the result will be accepted with risk [24].

$$
\begin{aligned}
& U=\left(r \sum \text { cations }-r \sum \text { anions } / r \sum \text { cations }+r \sum \text { anions }\right) \times 100 \\
& A=100-U
\end{aligned}
$$

Where; $\mathrm{U}$, is the uncertainty; $\mathrm{r}$ is a value in equivalent per mile (epm), and A is the certainty or accuracy.

As shown in Table (5), the ionic balance of nearly all and Raw samples are $<5 \%$. Which indicates that there is an electroneutrality, i.e the concentration of negative and positive ions is nearly similar with nearly $100 \%$ certainty $[22,25]$. So, the accuracy of the results could be used and dependent on it in hydrochemical interpretation [24].

\section{2- Measured TDS and Conductivity Ratio}

The $\mathrm{TDS}_{\mathrm{m}}$ - to - EC ratio could be used to determine the analyses' correctness and instruments' accuracy and the standard ratio ranges from 0.55 to 0.7 . If it is out, then either TDS or conductivity is suspect and needs to re-analyze [5]. The results in Table (5) reveals that all samples are within limit, i.e. accurate $\&$ accepted. 



\section{Table (5); ionic balance, accuracy and TDSm-to-EC ratio}

\begin{tabular}{|c|c|c|c|c|c|c|c|c|c|c|c|c|c|c|c|c|c|c|c|}
\hline Item & $1 \mathrm{~A}$ & 1B & $2 \mathrm{~A}$ & 2B & $3 \mathrm{~A}$ & 3B & $4 \mathrm{~A}$ & 4B & 5A & 5B & $6 \mathrm{~A}$ & $6 B$ & $7 \mathrm{~A}$ & 7B & $8 \mathrm{~A}$ & $8 B$ & 9A & 9B & $10 \mathrm{~A}$ \\
\hline$\Sigma$ Anions & 25.18 & 46.21 & 26.792 & 29.57 & 20.61 & 47.353 & 10.85 & 13.2 & 21.37 & 19.69 & 20.44 & 23.267 & 13.451 & 12.453 & 30.256 & 15.51 & 22.62 & 18.27 & 13.87 \\
\hline$\Sigma$ cations & 24.85 & 48.98 & 27.37 & 32.09 & 20.42 & 48.23 & 10.71 & 13.96 & 21.15 & 21.08 & 14.95 & 24.84 & \begin{tabular}{|l|}
13.3 \\
\end{tabular} & 13.4 & 30.98 & 14.74 & 28.35 & 18.09 & 14.95 \\
\hline$\Sigma$ Cat $+\Sigma$ Ani & 50.03 & 95.19 & 54.162 & 61.66 & 41.03 & 95.583 & 21.56 & 27.16 & 42.52 & 40.77 & 35.39 & 48.107 & 26.751 & 25.853 & 61.236 & 30.25 & 50.97 & 36.36 & 28.82 \\
\hline$\Sigma$ cat- $\Sigma$ Ani & -0.33 & 2.77 & 0.578 & 2.52 & -0.19 & 0.877 & -0.14 & 0.76 & -0.22 & 1.39 & -5.49 & 1.573 & -0.151 & 0.947 & 0.724 & -0.77 & 5.73 & -0.18 & 1.08 \\
\hline ionic balance & -0.0066 & 0.0291 & 0.0106 & 0.0409 & -0.005 & 0.009 & -0.006 & 0.028 & -0.0051 & 0.034 & -0.155 & 0.0327 & -0.005 & 0.037 & 0.012 & -0.025 & 0.112 & -0.005 & 0.037 \\
\hline $\mathbf{U}$ & -0.66 & 2.91 & 1.067 & 4.087 & -0.46 & 0.917 & -0.65 & 2.798 & -0.517 & 3.409 & -15.5 & 3.27 & -0.56 & 3.663 & 1.18 & -2.54 & 11.24 & -0.495 & 3.75 \\
\hline A & 100.6 & 97.1 & 98.9 & 95.9 & 100.46 & 99.08 & 100.65 & 97.20 & 100.51 & 96.59 & 115.5 & 96.73 & 100.56 & 96.34 & 98.81 & 102.5 & 88.76 & 100.5 & 96.25 \\
\hline TDSm/EC & 0.64 & 0.64 & 0.63 & 0.64 & 0.64 & 0.64 & 0.64 & 0.64 & 0.64 & 0.63 & 0.64 & 0.64 & 0.64 & 0.64 & 0.64 & 0.64 & 0.64 & 0.64 & 0.63 \\
\hline
\end{tabular}

Table (5) ; continued

\begin{tabular}{|c|c|c|c|c|c|c|c|c|c|c|c|c|c|c|c|c|c|c|c|}
\hline Item & $10 \mathrm{~B}$ & $11 \mathrm{~A}$ & $11 \mathrm{~B}$ & $12 \mathrm{~A}$ & $12 \mathrm{~B}$ & $13 \mathrm{~A}$ & $13 \mathrm{~B}$ & $14 \mathrm{~A}$ & $14 \mathrm{~B}$ & $15 \mathrm{~A}$ & $15 \mathrm{~B}$ & $16 \mathrm{~A}$ & $17 \mathrm{~A}$ & $18 \mathrm{~A}$ & $19 \mathrm{~A}$ & $19 \mathrm{~B}$ & $20 \mathrm{~A}$ & $20 \mathrm{~B}$ & Raw \\
\hline$\Sigma$ Anions & 28.44 & 20.79 & 24.16 & 16.69 & 27.41 & 39.71 & 42.42 & 49.97 & 39.49 & 13.86 & 15.86 & 21.98 & 12.464 & 11.87 & 11.42 & 17.81 & 10.21 & 14.15 & 47.01 \\
\hline$\Sigma$ cations & 30.39 & 20.48 & 25.89 & 16.39 & 27.04 & 39.64 & 39.32 & 46.58 & 37.78 & 14.09 & 15.9 & 22.39 & 12.31 & 11.72 & 11.49 & 19.08 & 10.14 & 15.07 & 42.93 \\
\hline$\Sigma$ ca $+\Sigma$ An & 58.83 & 41.27 & 50.05 & 33.08 & 54.45 & 79.35 & 81.74 & 96.55 & 77.27 & 27.955 & 31.763 & 44.37 & 24.774 & 23.59 & 22.914 & 36.892 & 20.353 & 29.219 & 89.94 \\
\hline$\Sigma$ ca- $\Sigma$ An & 1.95 & -0.31 & 1.73 & -0.3 & -0.37 & -0.07 & -3.1 & -3.39 & -1.71 & 0.225 & 0.037 & 0.41 & -0.154 & -0.15 & 0.066 & 1.268 & -0.073 & 0.921 & -4.08 \\
\hline ionic balance & 0.033 & -0.0075 & 0.0345 & -0.009 & -0.0068 & -0.0008 & -0.0379 & -0.0351 & -0.022 & 0.008 & 0.001 & 0.009 & -0.006 & -0.006 & 0.003 & 0.034 & -0.0036 & 0.0315 & -0.045 \\
\hline U & 3.31 & -0.75 & 3.45 & -0.9 & -0.68 & -0.08 & -3.79 & -3.51 & -2.2 & 0.80 & 0.1 & 0.9 & -0.6 & -0.6 & 0.3 & 3.4 & -0.36 & 3.15 & -4.5 \\
\hline A & 96.69 & 100.75 & 96.54 & 100.9 & 100.68 & 100.08 & 103.79 & 103.51 & 102.2 & 99.2 & 99.9 & 99.1 & 100.6 & 100.6 & 99.7 & 96.6 & 100.36 & 96.85 & 104.5 \\
\hline TDSm/EC & 0.64 & 0.64 & 0.64 & 0.64 & 0.64 & 0.6 & 0.65 & 0.65 & 0.65 & 0.64 & 0.64 & 0.64 & 0.64 & 0.64 & 0.64 & 0.64 & 0.64 & 0.64 & 0.61 \\
\hline
\end{tabular}

\subsection{Hydrochemical Properties}

1.Hydrochemical formula (Kurolov formula) and water type

Kurolov formula and water type was determined for results Tables (2, 4) using Eq. (1), as shown in Table (6). 



\section{Table (6): Hydrochemical formula}

\begin{tabular}{|c|c|c|c|c|c|c|c|c|c|c|c|}
\hline tem & $\begin{array}{c}\mathrm{Cl} \\
\mathrm{epm} \%\end{array}$ & $\begin{array}{l}\mathrm{HCO}_{3} \\
\mathrm{epm} \%\end{array}$ & \begin{tabular}{|c|}
$\mathrm{SO}_{4}$ \\
$\mathrm{epm} \%$
\end{tabular} & $\begin{array}{c}\mathrm{Ca} \\
\mathrm{epm} \%\end{array}$ & $\begin{array}{c}\mathbf{K} \\
\mathrm{epm} \%\end{array}$ & \begin{tabular}{c|c}
$\mathrm{Mg}$ \\
$\mathrm{epm} \%$
\end{tabular} & \begin{tabular}{c|c}
$\mathrm{Na}$ \\
$\mathrm{epm} \%$
\end{tabular} & Hydrochemical Formula & Water type & $\begin{array}{c}\text { Pred. } \\
\text { salts }\end{array}$ & No. \& \% \\
\hline $1 \mathrm{~A}$ & 66.3 & 17.9 & 15.7 & 15.3 & 2.21 & 8.45 & 74 & $\begin{array}{c}1625(\mathrm{Cl}(66.3 \%) \mathrm{HCO}(17.9 \%) \mathrm{SO}(15 . \\
\mathrm{Na}(74 \%) \mathrm{Ca}(15.3 \%) 8.3\end{array}$ & $\begin{array}{l}\text { (Ca-Sodium-SO }{ }_{4}-\mathrm{HCO}_{3}- \\
\text { Chloride) }\end{array}$ & $\mathrm{NaCl}$ & (1)2.7 \\
\hline $8 \mathrm{~A}$ & 65.7 & 12.84 & 21.4 & 24.85 & 0.26 & 11.62 & 63.26 & $\begin{array}{l}1997(\mathrm{Cl}(65.7 \%) \text { SO4(21.4\%) } \\
/ \mathrm{Na}(63.27 \%) \mathrm{Ca}(24.8 \%) 7.3\end{array}$ & (Ca-Sodium-SO 4 -Chloride) & $\mathrm{NaCl}$ & (1)2.7 \\
\hline 1B & 80.7 & 6.41 & 12.9 & 18.7 & 0.26 & 2.45 & 78.6 & $\begin{array}{l}3187 \mathrm{Cl}(80.7 \%) \\
/ \mathrm{Na}(78.6 \%) \mathrm{Ca}(18.7 \%) 7.75\end{array}$ & (Ca-Sodium-Chloride) & $\mathrm{NaCl}$ & $\begin{array}{ll}(6) \\
16.21\end{array}$ \\
\hline 3B & 84.5 & 8.3 & 7.18 & 17.73 & 0.41 & 9.7 & 72.15 & $\begin{array}{l}3283(\mathrm{Cl}(84.5 \%) \\
/ \mathrm{Na}(72.1 \%) \mathrm{Ca}(17.7 \%) 7.55\end{array}$ & (Ca-Sodium-Chloride) & $\mathrm{NaCl}$ & \\
\hline $13 \mathrm{~A}$ & 82.6 & 4.71 & 12.68 & 20.18 & 0.3 & 12.66 & 66.85 & $\begin{array}{l}2400(\mathrm{Cl}(82.6 \%) \\
/ \mathrm{Na}(66.8 \%) \mathrm{Ca}(20.2 \%)) 76.5\end{array}$ & (Ca-Sodium-Chloride) & $\mathrm{NaCl}$ & \\
\hline 13B & 84.9 & 5.76 & 9.29 & 19.45 & 0.38 & 12.77 & 67.4 & $\begin{array}{l}2707(\mathrm{Cl}(84.9 \%) \\
/ \mathrm{Na}(67.4 \%) \mathrm{Ca}(19.4 \%) 7.9\end{array}$ & (Ca-Sodium-Chloride) & $\mathrm{NaCl}$ & \\
\hline $14 \mathrm{~A}$ & 83.2 & 2.83 & 13.89 & 19.64 & 0.28 & 12.88 & 67.2 & $\begin{array}{l}3280(\mathrm{Cl}(83.2 \%) \\
/ \mathrm{Na}(67.2 \%) \mathrm{Ca}(19.6 \%) 7.7\end{array}$ & (Ca-Sodium-Chloride) & $\mathrm{NaCl}$ & \\
\hline 14B & 83.6 & 6.33 & 10 & 18 & 0.4 & 10.67 & 70.94 & $\begin{array}{l}2652(\mathrm{Cl}(83.6 \%) \\
/ \mathrm{Na}(70.9 \%) \mathrm{Ca}(18 \%)) 7.8\end{array}$ & (Ca-Sodium-Chloride) & $\mathrm{NaCl}$ & \\
\hline $2 \mathrm{~A}$ & 78.4 & 9.2 & 12.4 & 13.1 & 0.29 & 7.27 & 79.3 & $1720(\mathrm{Cl}(78.4 \%) / \mathrm{Na}(79.3 \%) 8.4$ & (Sodium-Chloride) & $\mathrm{NaCl}$ & (4) \\
\hline $9 \mathrm{~A}$ & 74.7 & 13 & 12.27 & 11.64 & 0.46 & 6.07 & 81.83 & $1829(\mathrm{Cl}(74.7 \%) / \mathrm{Na}(81.8 \%)) 7.67$ & (Sodium-Chloride) & $\mathrm{NaCl}$ & 10.81 \\
\hline 11B & 77. & & 9.5 & 12.4 & 0.7 & 5.4 & 81.5 & $1669(\mathrm{Cl}(77.2 \%) / \mathrm{Na}(81.5 \%)) 7.05$ & (Sodium-Chloride) & $\mathrm{NaCl}$ & \\
\hline 12B & 77.5 & 14.55 & 7.93 & 8.91 & 0.55 & 10.28 & 80.25 & $1881(\mathrm{Cl}(77.5 \%) / \mathrm{Na}(80.2 \%)) 7.74$ & (Sodium-Chloride) & $\mathrm{NaCl}$ & \\
\hline 2B & 78.8 & 15.5 & 5.67 & 11.62 & 0.4 & 3.83 & 84.1 & $\begin{array}{r}2112(\mathrm{Cl}(78.8 \%) H C O 3(15.5 \%) \\
/ \mathrm{Na}(84.1 \%) 7.71\end{array}$ & (Sodium-HCO 3 -Chloride) & $\mathrm{NaCl}$ & $\begin{array}{l}(19) \\
51.35\end{array}$ \\
\hline $3 \mathbf{A}$ & 77.7 & 18.2 & 4.09 & 11.5 & 0.49 & 9.1 & 78.8 & $\begin{array}{r}1231(\mathrm{Cl}(77.7 \%) H \operatorname{CO3}(18.2 \%) \\
/ \mathrm{Na}(78.8 \%) 8.3\end{array}$ & (Sodium-HCO 3 -Chloride) & $\mathrm{NaCl}$ & \\
\hline $4 \mathrm{~A}$ & 54.4 & 41.3 & 4.23 & 7.93 & 0.75 & 10.1 & 81.23 & $\begin{array}{r}663(\mathrm{Cl}(54.4 \%) H \operatorname{CO3}(41.3 \%) \\
/ \mathrm{Na}(81.2 \%) 8.4\end{array}$ & (Sodium-HCO 3 -Chloride) & $\mathrm{NaCl}$ & \\
\hline 4B & 67.1 & 28.8 & 4.06 & 7.16 & 0.71 & 4.73 & 87.39 & $\begin{array}{r}925(\mathrm{Cl}(67.1 \%) H \operatorname{CO3}(28.8 \%) \\
\quad / \mathrm{Na}(87.4 \%)) 8.34\end{array}$ & (Sodium-HCO 3 -Chloride) & $\mathrm{NaCl}$ & \\
\hline 5B & 72.4 & 18.8 & 8.72 & 14.2 & 0.71 & 5.83 & 79.2 & $\begin{array}{r}1641(\mathrm{Cl}(72.4 \%) H C O 3(18.8 \%) \\
/ \mathrm{Na}(79.2 \%) 7.59\end{array}$ & (Sodium-HCO 3 -Chloride) & $\mathrm{NaCl}$ & \\
\hline $6 \mathrm{~A}$ & 66.3 & 27.1 & 6.5 & 11.37 & 0.94 & 5.42 & 82.3 & $\begin{array}{r}1114(\mathrm{Cl}(66.3 \%) H C O 3(27.1 \%) \\
/ \mathrm{Na}(82.3 \%) 8.4\end{array}$ & (Sodium-HCO 3 -Chloride) & $\mathrm{NaCl}$ & \\
\hline $7 \mathrm{~A}$ & 58.5 & 39.6 & 1.86 & 5.41 & 0.6 & 6.01 & 87.97 & $\begin{array}{r}846(\mathrm{Cl}(58.5 \%) H C O 3(339.6 \%) \\
/ \mathrm{Na}(87.97 \%)) 7.85\end{array}$ & (Sodium-HCO 3 -Chloride) & $\mathrm{NaCl}$ & \\
\hline $7 \mathrm{~B}$ & 58.8 & 37.51 & 3.69 & 9.18 & 0.74 & 8.73 & 81.34 & $\begin{array}{r}891(\mathrm{Cl}(58.8 \%) H C O 3(37.5 \%) \\
/ \mathrm{Na}(81.3 \%)) 8.02 \\
\end{array}$ & (Sodium-HCO 3 -Chloride) & $\mathrm{NaCl}$ & \\
\hline 8B & 67.7 & 27.31 & 4.96 & 8.82 & 0.54 & 4.48 & 86.16 & \begin{tabular}{|}
$\begin{array}{r}996(\mathrm{Cl}(67.7 \%) H C O 3(27.3 \%) \\
/ \mathrm{Na}(86.16 \%)) 7.88\end{array}$ \\
\end{tabular} & (Sodium-HCOy-Chloride) & $\mathrm{NaCl}$ & \\
\hline 9R & 58.8 & 36.9 & 4.28 & 10.37 & 1.2 & 5.48 & 82.94 & $\begin{array}{r}940(\mathrm{CI}(58.8 \%) H \operatorname{CO3}(36.9 \%) \\
\qquad / \mathrm{Na}(82.9 \%)) 7.75\end{array}$ & (Sodium-HCO - -Chloride) & $\mathrm{NaCl}$ & \\
\hline $10 \mathrm{~A}$ & 63.2 & 29.64 & 7.11 & 14.92 & 0.33 & 6.24 & 78.51 & $\begin{array}{r}1104(\mathrm{Cl}(63.2 \%) H C O 3(29.64 \%) \\
/ \mathrm{Na}(78.5 \%)) 8.39\end{array}$ & (Sodium-HCO 3 -Chloride) & $\mathrm{NaCl}$ & \\
\hline 10B & 76.9 & 14.99 & 8.08 & 13.26 & 0.43 & 4.05 & 82.26 & $1910(\mathrm{Cl}(76.9 \%) \mathrm{HCO3(}$ & (Sodium-HCO 3 -Chloride) & $\mathrm{NaCl}$ & \\
\hline $15 \mathrm{~A}$ & 66.5 & 24.22 & 9.23 & 10.64 & 0.71 & 2.06 & 86.6 & $\begin{array}{r}943(\mathrm{Cl}(66.5 \%) \mathrm{HCO3}(24 \\
/ \mathrm{Na}(8\end{array}$ & (Sodium-HCO ${ }_{3}$-Chloride) & $\mathrm{NaCl}$ & \\
\hline $17 \mathrm{~A}$ & 56.1 & 38.12 & 5.7 & 8.28 & 0.4 & 6.82 & 84.48 & $\begin{array}{r}821(\mathrm{Cl}(56.1 \%) H C O 3(38.1 \%) \\
/ \mathrm{Na}(84.5 \%)) 7.94\end{array}$ & (Sodium-HCO 3 -Chloride) & $\mathrm{NaCl}$ & \\
\hline $18 \mathrm{~A}$ & 64.4 & 29.63 & 5.96 & 12.46 & 0.43 & 11.43 & 75.68 & $\begin{array}{c}740(\mathrm{Cl}(64.4 \%) H \operatorname{CO}(29.6 \%) \\
/ \mathrm{Na}(75.7 \%)) 8.38 \\
\end{array}$ & (Sodium-HCO ${ }_{3}$-Chloride) & $\mathrm{NaCl}$ & \\
\hline $19 \mathrm{~A}$ & 61.4 & 32.14 & 6.43 & 12.01 & 0.26 & 12.01 & 75.72 & $\begin{array}{r}749(\mathrm{Cl}(61.4 \%) \mathrm{HCO3}(32.14 \%) \\
/ \mathrm{Na}(75.7 \%)) 8.46\end{array}$ & (Sodium-HCO 3 -Chloride) & $\mathrm{NaCl}$ & \\
\hline 19B & 67.4 & 25.32 & 7.3 & 9.17 & 0.42 & 6.03 & 84.38 & $\begin{array}{r}1224(\mathrm{Cl}(67.4 \%) H C O 3(25.3 \%) \\
/ \mathrm{Na}(84.4 \%)) 7.89\end{array}$ & (Sodium-HCO 3 -Chloride) & $\mathrm{NaCl}$ & \\
\hline $20 \mathrm{~A}$ & 44.2 & 43.56 & 12.17 & 12.82 & 0.3 & 11.93 & 74.95 & $\begin{array}{r}643(\mathrm{Cl}(44.2 \%) H C O 3(43.56 \%) \\
/ \mathrm{Na}(74.9 \%)) 8.33\end{array}$ & (Sodium-HCO 3 -Chloride) & $\mathrm{NaCl}$ & \\
\hline 20B & 67.1 & 26.8 & 6.01 & 10.95 & 0.53 & 4.91 & 83.61 & $\begin{array}{r}966(\mathrm{Cl}(67.1 \%) \mathrm{HCO3}(26.8 \%) \\
/ \mathrm{Na}(83.6 \%)) 7.6\end{array}$ & (Sodium-HCO3-Chloride) & $\mathrm{NaCl}$ & \\
\hline $5 \mathrm{~A}$ & 68.6 & 19.8 & 11.6 & 15.41 & 0.38 & 6.19 & 78 & $\begin{array}{l}1319(\mathrm{Cl}(68.6 \%) \mathrm{HCO3}(19.8 \%) \\
/ \mathrm{Na}(78 \%) \mathrm{Ca}(15.4 \%)) 8.37\end{array}$ & $\begin{array}{c}\text { (Ca-Sodium-HCO3- } \\
\text { Chloride) }\end{array}$ & $\mathrm{NaCl}$ & $\begin{array}{c}(6+\text { raw }) \\
16.21\end{array}$ \\
\hline 6B & 69.8 & 15.2 & 14.92 & 15.5 & 1.13 & 7.29 & 76.1 & $\begin{array}{l}1651(\mathrm{Cl}(69.8 \%) \mathrm{HCO3}(15.2 \%) \\
/ \mathrm{Na}(76.1 \%) \mathrm{Ca}(15.5 \%) 7.61\end{array}$ & $\begin{array}{l}\text { (Ca-Sodium-HCO3- } \\
\text { Chloride) }\end{array}$ & $\mathrm{NaCl}$ & \\
\hline $11 \mathrm{~A}$ & 69.97 & 19.92 & 10.1 & 18.65 & 0.63 & 6.49 & 74.22 & $\begin{array}{l}1356(\mathrm{Cl}(69.97 \%) \mathrm{HCO3}(19.92 \%) \\
/ \mathrm{Na}(74.2 \%) \mathrm{Ca}(18.65 \%)) 7.89\end{array}$ & $\begin{array}{c}\text { (Ca-Sodium-HCO3- } \\
\text { Chloride) }\end{array}$ & $\mathrm{NaCl}$ & \\
\hline $12 \mathrm{~A}$ & 64.5 & 23.7 & 11.76 & 18.97 & 0.43 & 13.48 & 67.11 & $\begin{array}{l}1081(\mathrm{Cl}(64.5 \%) \mathrm{HCO3}(23.7 \%) \\
/ \mathrm{Na}(67.1 \%) \mathrm{Ca}(18.97 \%)) 7.81\end{array}$ & $\begin{array}{c}\text { (Ca-Sodium-HCO3- } \\
\text { Chloride) }\end{array}$ & $\mathrm{NaCl}$ & \\
\hline 15B & 60.5 & 33.67 & 5.8 & 16.35 & 0.63 & 6.29 & 76.73 & $\begin{array}{l}1182(\mathrm{Cl}(60.5 \%) \mathrm{HCO}(33.67 \%) \\
/ \mathrm{Na}(76.73 \%) \mathrm{Ca}(16.3 \%)) 7.87\end{array}$ & $\begin{array}{c}\text { (Ca-Sodium-HCO3- } \\
\text { Chloride) }\end{array}$ & $\mathrm{NaCl}$ & \\
\hline $16 \mathrm{~A}$ & 69 & 17.71 & 13.25 & 24.56 & 0.45 & 14.69 & 60.3 & $\begin{array}{l}1404(\mathrm{Cl}(69 \%) \mathrm{HCO3}(17.7 \%) \\
/ \mathrm{Na}(60.3 \%) \mathrm{Ca}(24.5 \%) 7.45\end{array}$ & $\begin{array}{c}\text { (Ca-Sodium-HCO3- } \\
\text { Chloride) }\end{array}$ & $\mathrm{NaCl}$ & \\
\hline Raw & 70.8 & 17.91 & 11.26 & 27.72 & 2.37 & 9.57 & 60.33 & $\begin{array}{c}2858(\mathrm{Cl}(70.8 \%) \mathrm{HCO3}(179 \%) / \\
\mathrm{Na}(60.3 \%) \mathrm{Ca}(27.7 \%)) 7.38^{*}\end{array}$ & $\begin{array}{c}\text { (Ca-Sodium-HCO3- } \\
\text { Chloride) }\end{array}$ & $\mathrm{NaCl}$ & \\
\hline
\end{tabular}



Kurolov formula showed that there are six hydrochemical formula in the study area and the predominant salt is $\mathrm{NaCl}$, sodium chloride water type, as shown in Table (7) the six hydrochemical formula are:
Sodium-HCO${ }_{3}^{-}$Chloride
$\mathrm{Ca}$-Sodium-HCO $\mathrm{C}_{3}$-Chloride
$51.35 \%$
Ca-Sodium- Chloride
Sodium-Chloride10.81\%
$\mathrm{Ca}$-Sodium-SO $\mathrm{S}_{4} \mathrm{HCO}_{3}$-Chloride

\section{$16.21 \%$}
$16.21 \%$
Ca-Sdium-SO $\mathrm{O}_{4}$-Chloride
$2.7 \%$
$2.7 \%$

\section{Hypothetical Salts}

Water hypothetical salts for results in Table(4) were calculated by correlation between anions and cations Table(7).

Table (7); Hypothetical salts for water samples of study area

\begin{tabular}{|c|c|c|c|c|c|c|c|c|c|c|c|c|c|c|c|c|c|c|c|c|}
\hline Hypothetical salts & 1A & 1B & $\mathbf{2 A}$ & 2B & $3 \mathbf{A}$ & 3B & $4 \mathrm{~A}$ & 4B & 5A & 5B & 6A & 6B & $7 \mathbf{A}$ & $7 \mathbf{B}$ & $\mathbf{8 A}$ & 8B & 9A & 9B & $10 \mathrm{~A}$ & 10B \\
\hline $\mathrm{KCl}$ & 2.21 & 0.26 & 0.29 & 0.4 & 0.49 & 0.41 & 0.75 & 0.71 & 0.38 & 0.71 & 0.94 & 1.13 & 0.6 & 0.74 & 0.26 & 0.54 & 0.46 & 1.2 & 0.33 & 0.43 \\
\hline $\mathrm{NaCl}$ & 64.09 & 78.6 & 78.11 & 78.4 & 77.21 & 72.15 & 53.65 & 66.39 & 68.22 & 76.69 & 65.36 & 68.67 & 57.9 & 58.06 & 63.26 & 67.16 & $\mathbf{7 4 . 2 4}$ & 57.6 & 62.87 & 76.47 \\
\hline $\mathrm{MgCl}_{2}$ & 0.0 & 1.84 & 0.0 & 0.0 & 0.0 & \begin{tabular}{|l|l}
9.7 & \\
\end{tabular} & 0.0 & 0.0 & 0.0 & 0.0 & 0.0 & 0.0 & 0.0 & 0.0 & 2.18 & 0.0 & 0.0 & 0.0 & 0.0 & 0.0 \\
\hline $\mathrm{CaCl}_{2}$ & 0.0 & 0.0 & 0.0 & 0.0 & 0.0 & 2.24 & 0.0 & 0.0 & 0.0 & 0.0 & 0.0 & 0.0 & 0.0 & 0.0 & 0.0 & 0.0 & 0.0 & 0.0 & 0.0 & 0.0 \\
\hline $\mathrm{K}_{2} \mathrm{SO}_{4}$ & 0.0 & 0.0 & 0.0 & 0.0 & 0.0 & 0.0 & 0.0 & 0.0 & 0.0 & 0.0 & 0.0 & 0.0 & 0.0 & 0.0 & 0.0 & 0.0 & 0.0 & 0.0 & 0.0 & 0.0 \\
\hline $\mathrm{Na}_{2} \mathrm{SO}_{4}$ & 9.91 & 0.0 & 1.19 & 5.67 & 1.59 & 0.0 & 4.23 & 4.06 & 9.78 & 2.51 & 6.5 & 7.43 & 1.86 & 3.69 & 0.0 & 4.96 & 7.59 & 4.28 & 7.11 & 5.79 \\
\hline $\mathrm{Mg} \mathrm{SO}_{4}$ & 5.79 & 0.61 & 11.21 & 0.0 & 2.5 & 0.0 & 0.0 & 0.0 & 1.82 & 5.83 & 0.0 & 7.29 & 0.0 & 0.0 & 9.44 & 0.0 & 4.68 & 0.0 & 0.0 & 2.29 \\
\hline $\mathrm{CaSO}_{4}$ & 0.0 & 12.29 & 0.0 & 0.0 & 0.0 & 7.18 & 0.0 & 0.0 & 0.0 & 0.38 & 0.0 & 0.2 & 0.0 & 0.0 & 11.96 & 0.0 & 0.0 & 0.0 & 0.0 & 0.0 \\
\hline $\mathrm{NaHCO}_{3}$ & 0.0 & 0.0 & 0.0 & 0.03 & 0.0 & 0.0 & 23.35 & 16.94 & 0.0 & 0.0 & 10.44 & 0.0 & 28.21 & 19.59 & 0.0 & 14.04 & 0.0 & 21.06 & 8.53 & 0.0 \\
\hline $\mathrm{Mg}\left(\mathrm{HCO}_{3}\right)_{2}$ & 2.66 & 0.0 & 0.0 & 3.83 & 6.6 & 0.6 & 10.1 & 4.73 & 4.37 & 0.0 & 5.42 & 0.0 & 6.01 & 8.73 & 0.0 & 4.48 & 1.39 & 5.48 & 6.24 & 1.76 \\
\hline $\mathrm{Ca}\left(\mathrm{HCO}_{3}\right)_{2}$ & 15.24 & 6.41 & 5.26 & 11.62 & 11.5 & 8.3 & 7.85 & 7.13 & 15.41 & 13.82 & 11.24 & 15.2 & 5.38 & 9.18 & 12.84 & 8.79 & 11.61 & 10.36 & 14.87 & 13.23 \\
\hline
\end{tabular}

\section{Table (7): continued}

\begin{tabular}{|c|c|c|c|c|c|c|c|c|c|c|c|c|c|c|c|c|c|c|c|}
\hline Hypothetical salts & $10 \mathrm{~B}$ & $11 \mathrm{~A}$ & 11B & $12 \mathrm{~A}$ & 12B & 13A & 13B & $14 \mathrm{~A}$ & 14B & 15A & 15B & $16 \mathrm{~A}$ & 17A & 18A & 19A & 19B & $20 A$ & $20 B$ & Raw* \\
\hline KCl & 0.43 & 0.63 & 0.7 & 0.43 & 0.55 & 0.3 & 0.38 & 0.28 & 0.4 & 0.71 & 0.63 & 0.45 & 0.4 & 0.43 & 0.26 & 0.42 & 0.3 & 0.53 & 2.37 \\
\hline $\mathrm{NaCl}$ & 76.47 & 69.34 & 76.5 & 64.07 & 76.95 & 66.85 & 67.4 & 67.2 & 70.94 & 65.79 & 59.87 & 60.3 & 55.7 & 63.97 & 61.14 & 66.98 & 43.9 & 66.57 & 60.33 \\
\hline $\mathrm{MgCl}_{2}$ & 0.0 & 0.0 & $\mathbf{0 . 0}$ & 0.0 & 0.0 & 12.66 & 12.77 & 12.88 & 10.67 & 0.0 & 0.0 & 8.25 & 0.0 & 0.0 & 0.0 & 0.0 & 0.0 & 0.0 & 8.1 \\
\hline $\mathrm{CaCl}_{2}$ & 0.0 & 0.0 & 0.0 & 0.0 & 0.0 & 2.79 & 4.35 & 2.84 & 1.59 & 0.0 & 0.0 & 0.0 & 0.0 & 0.0 & 0.0 & 0.0 & 0.0 & 0.0 & 0.0 \\
\hline $\mathrm{K}_{2} \mathrm{SO}_{4}$ & 0.0 & 0.0 & 0.0 & 0.0 & 0.0 & 0.0 & 0.0 & 0.0 & 0.0 & 0.0 & 0.0 & 0.0 & 0.0 & 0.0 & 0.0 & 0.0 & 0.0 & 0.0 & 0.0 \\
\hline $\mathrm{Na}_{2} \mathrm{SO}_{4}$ & 5.79 & 4.88 & 5.0 & 3.04 & 3.3 & 0.0 & 0.0 & 0.0 & 0.0 & 9.23 & 5.8 & 0.0 & 5.7 & 5.96 & 6.43 & 7.3 & 12.17 & 6.01 & 0.0 \\
\hline $\mathrm{Mg} \mathrm{SO}_{4}$ & 2.29 & 5.22 & 4.5 & 8.72 & 4.63 & 0.0 & 0.0 & 0.0 & 0.0 & 0.0 & 0.0 & 6.44 & 0.0 & 0.0 & 0.0 & 0.0 & 0.0 & 0.0 & 1.47 \\
\hline $\mathrm{CaSO}_{4}$ & 0.0 & 0.0 & 0.0 & 0.0 & 0.0 & 12.68 & 9.29 & 13.89 & 10 & 0.0 & 0.0 & 6.81 & 0.0 & 0.0 & 0.0 & 0.0 & 0.0 & 0.0 & 9.79 \\
\hline $\mathrm{NaHCO}_{3}$ & 0.0 & 0.0 & 0.0 & 0.0 & 0.0 & 0.0 & 0.0 & 0.0 & 0.0 & 11.58 & 11.06 & 0.0 & 23.08 & 5.75 & 8.15 & 10.01 & 18.88 & 11.03 & 0.0 \\
\hline $\mathrm{Mg}\left(\mathrm{HCO}_{3}\right)_{2}$ & 1.76 & 1.27 & 0.9 & 4.76 & 5.65 & 0.0 & 0.0 & 0.0 & 0.0 & 2.06 & 6.29 & 0.0 & 6.82 & 11.43 & 12.01 & 6.03 & 11.93 & 4.91 & 0.0 \\
\hline $\mathrm{Ca}\left(\mathrm{HCO}_{3}\right)_{2}$ & 13.23 & 18.65 & 12.4 & 18.94 & 8.9 & 4.71 & 5.76 & 2.83 & 6.33 & 10.58 & 16.32 & 17.71 & 8.22 & 12.45 & 11.98 & 9.17 & 12.75 & 10.86 & 17.91 \\
\hline
\end{tabular}



The combination between major anions and cations reveals the formation of seven assemblages of hypothetical salts combinations as shown in Tables $(7,8)$. About $45.95 \%$ of the groundwater samples are characterized by assemblage I, about $24.3 \%$ are characterized by assemblage II, and about $10.8 \%$ characterized by assemblage III and about $8.1 \%$ and raw sample are characterized by assemblage IV. They are characterized by the presence of $\mathrm{NaCl}$, $\mathrm{Na}_{2} \mathrm{SO}_{4}, \mathrm{NaHCO}_{3}, \mathrm{Mg}\left(\mathrm{HCO}_{3}\right)_{2}$ and $\mathrm{Ca}\left(\mathrm{HCO}_{3}\right)_{2} ; \mathrm{NaCl}, \mathrm{Na}_{2} \mathrm{SO}_{4}$, $\mathrm{MgSO}_{4}, \mathrm{Mg}\left(\mathrm{HCO}_{3}\right)_{2}$ and $\mathrm{Ca}\left(\mathrm{HCO}_{3}\right)_{2} ; \mathrm{NaCl}, \mathrm{MgCl}_{2}, \mathrm{CaCl}_{2}, \mathrm{CaSO}_{4}$, and $\mathrm{Ca}\left(\mathrm{HCO}_{3}\right)_{2}$ and; $\mathrm{NaCl}, \quad \mathrm{MgCl}_{2}, \mathrm{MgSO}_{4}, \mathrm{CaSO}_{4}$, and $\mathrm{Ca}\left(\mathrm{HCO}_{3}\right)_{2}$ salts respectively. Assemblages $\mathrm{V}$ characterize about $5.4 \%$ of the groundwater samples is characterized by the presence of $\mathrm{NaCl}, \mathrm{Na}_{2} \mathrm{SO}_{4}, \mathrm{MgSO}_{4}, \mathrm{CaSO}_{4}$ and $\mathrm{Ca}\left(\mathrm{HCO}_{3}\right)_{2}$. Finally; each of assemblages VI and VII characterize about $2.7 \%$ of samples are characterized by hypothetical salt assemblage (III) $\mathrm{NaCl}, \mathrm{MgCl}_{2}$, $\mathrm{CaCl}_{2}, \mathrm{CaSO}_{4}, \mathrm{Mg}\left(\mathrm{HCO}_{3}\right)_{2}$ and $\mathrm{Ca}\left(\mathrm{HCO}_{3}\right)_{2}$ and; $\mathrm{NaCl}, \mathrm{Na}_{2} \mathrm{SO}_{4}$, $\mathrm{MgSO}_{4}$, and $\mathrm{Ca}\left(\mathrm{HCO}_{3}\right)_{2}$ respectively. Assemblages $\mathrm{NaCl}, \mathrm{MgCl}_{2}$, $\mathrm{CaCl}_{2}, \mathrm{CaSO}_{4}$, and $\mathrm{Ca}\left(\mathrm{HCO}_{3}\right)_{2}$, in water samples $13 \mathrm{~A}, 13 \mathrm{~B}, 14 \mathrm{~A}$ and14B (localized in east side of the site) which is similar to that of Nile water and forms only $10.8 \%$, which confirms that the recharge from River Nile in this region is very week .Generally, the aquifer in this region is not belongs to the river Nile aquifer, Table (8).The presence of marine salts of $\mathrm{NaCl}, \mathrm{Na}_{2} \mathrm{SO}_{4}$ and $\mathrm{MgSO}_{4}$ and $\mathrm{CaSO}_{4}$ is may be due to the flushing of salt water by fresh water through local heavy infiltration of rainwater in the past pluvial times. The longterm contact time between rock matrix and water also, due to the dissolution of these salts encountered in the quaternary and pliocene water bearing sediments [11]. 
Table (8); Assemblages of hypothetical salts for wells and raw water samples of study area

\begin{tabular}{|c|c|c|c|c|}
\hline $\begin{array}{l}\text { Assemblages of hypothetical } \\
\text { salts combinations }\end{array}$ & $\begin{array}{l}\text { Classifi } \\
\text {-cation }\end{array}$ & Sample no. & Number & $\%$ \\
\hline $\begin{array}{l}\mathrm{NaCl}, \mathrm{Na} 2 \mathrm{SO} 4, \mathrm{NaHCO} \\
\mathrm{Mg}(\mathrm{HCO} 3) 2, \mathrm{Ca}(\mathrm{HCO3}) 2\end{array}$ & I & $\begin{array}{c}\text { 2B, 4A, 4B, 6A, 7A, 7B, 8B, 9B, 10A, } \\
\text { 15A, 15B, 17A, 18A, 19A, 19B, 20A, 20B }\end{array}$ & 17 & 45.95 \\
\hline $\begin{array}{l}\mathrm{NaCl}, \mathrm{Na} 2 \mathrm{SO} 4, \mathrm{Mg} \mathrm{SO} 4 \\
\mathrm{Mg}(\mathrm{HCO}) 2, \mathrm{Ca}(\mathrm{HCO3}) 2\end{array}$ & II & $1 \mathrm{~A}, 3 \mathrm{~A}, 5 \mathrm{~A}, 9 \mathrm{~A}, 10 \mathrm{~B}, 11 \mathrm{~A}, 11 \mathrm{~B}, 12 \mathrm{~A}, 12 \mathrm{~B}$ & 9 & 24.3 \\
\hline $\begin{array}{l}\mathrm{NaCl}, \mathrm{MgCl} 2, \mathrm{CaCl} 2 \\
\mathrm{CaSO} 4, \mathrm{Ca}(\mathrm{HCO3}) 2\end{array}$ & III & $\begin{array}{c}\text { 13A, 13B, 14A, 14B, (localized in East } \\
\text { side) }\end{array}$ & 4 & 10.8 \\
\hline $\begin{array}{l}\mathrm{NaCl}, \mathrm{MgCl} 2, \mathrm{Mg} \mathrm{SO} 4 \\
\mathrm{CaSO} 4, \mathrm{Ca}(\mathrm{HCO3}) 2\end{array}$ & IV & $1 \mathrm{~B}, 8 \mathrm{~A}, 16 \mathrm{~A}$, and $($ Raw $)$ & 3 & 8.1 \\
\hline $\begin{array}{l}\mathrm{NaCl}, \mathrm{Na} 2 \mathrm{SO} 4, \mathrm{Mg} \mathrm{SO} 4 \\
\quad \mathrm{CaSO} 4, \mathrm{Ca}(\mathrm{HCO3}) 2\end{array}$ & $\mathbf{V}$ & $5 B, 6 B$ & 2 & 5.4 \\
\hline $\begin{array}{c}\mathrm{NaCl}, \mathrm{MgCl}, \mathrm{CaCl}, \\
\mathrm{CaSO}, \\
\mathrm{Mg}(\mathrm{HCO3}) 2, \mathrm{Ca}(\mathrm{HCO3}) 2,\end{array}$ & VI & 3B & 1 & 2.7 \\
\hline $\begin{array}{l}\mathrm{NaCl}, \mathrm{Na} 2 \mathrm{SO} 4, \mathrm{Mg} \mathrm{SO} 4 \\
\mathrm{Ca}(\mathrm{HCO}) 2\end{array}$ & VII & $2 \mathrm{~A}$ & 1 & 2.7 \\
\hline $\begin{array}{l}\mathrm{NaCl}, \mathrm{Na}_{2} \mathrm{SO}_{4}, \mathrm{Mg} \mathrm{SO}_{4} \\
\mathrm{Mg}\left(\mathrm{HCO}_{3}\right)_{2}, \mathrm{Ca}\left(\mathrm{HCO}_{3}\right)_{2}\end{array}$ & II & $1 \mathrm{~A}, 3 \mathrm{~A}, 5 \mathrm{~A}, 9 \mathrm{~A}, 10 \mathrm{~B}, 11 \mathrm{~A}, 11 \mathrm{~B}, 12 \mathrm{~A}, 12 \mathrm{~B}$ & 9 & 24.3 \\
\hline $\begin{array}{c}\mathrm{NaCl}, \mathrm{MgCl}_{2}, \mathrm{CaCl}_{2} \\
\mathrm{CaSO}_{4}, \mathrm{Ca}\left(\mathrm{HCO}_{3}\right)_{2}\end{array}$ & III & $\begin{array}{c}\text { 13A, 13B, 14A, 14B, (localized in East } \\
\text { side) }\end{array}$ & 4 & 10.8 \\
\hline $\begin{array}{c}\mathrm{NaCl}, \mathrm{MgCl}_{2}, \mathrm{Mg} \mathrm{SO}_{4} \\
\mathrm{CaSO}_{4}, \mathrm{Ca}\left(\mathrm{HCO}_{3}\right)_{2}\end{array}$ & IV & 1B, 8A, 16A, and (Raw) & 3 & 8.1 \\
\hline $\begin{array}{l}\mathrm{NaCl}, \mathrm{Na}_{2} \mathrm{SO}_{4}, \mathrm{Mg} \mathrm{SO}_{4}, \\
\quad \mathrm{CaSO}_{4}, \mathrm{Ca}\left(\mathrm{HCO}_{3}\right)_{2}\end{array}$ & $\mathbf{V}$ & $5 B, 6 B$ & 2 & 5.4 \\
\hline $\begin{array}{c}\mathrm{NaCl}, \mathrm{MgCl}_{2}, \mathrm{CaCl}_{2} \\
\mathrm{CaSO}_{4}, \\
\mathrm{Mg}\left(\mathrm{HCO}_{3}\right)_{2}, \mathrm{Ca}\left(\mathrm{HCO}_{3}\right)_{2}\end{array}$ & VI & 3B & 1 & 2.7 \\
\hline $\begin{array}{c}\mathrm{NaCl}, \mathrm{Na}_{2} \mathrm{SO}_{4}, \mathrm{Mg} \mathrm{SO}_{4}, \\
\mathrm{Ca}\left(\mathrm{HCO}_{3}\right)_{2}\end{array}$ & VII & $\mathbf{2 A}$ & 1 & 2.7 \\
\hline
\end{tabular}

\section{Hydrochemical Indicators}

Hydrochemical indicators ratios between the different major ions, Table (5), as; $\mathrm{rCa} / \mathrm{rCl}, \mathrm{rCa} / \mathrm{rMg}, \mathrm{rMg} / \mathrm{rCl}, \mathrm{rNa} / \mathrm{rCl}, \mathrm{rK} / \mathrm{rCl}$, $\mathrm{rSO}_{4} / \mathrm{rCl}, \mathrm{rNa}+\mathrm{rK} / \mathrm{rCl}, \mathrm{rHCO}_{3} / \mathrm{rCl}$ and $(\mathrm{rNa}+\mathrm{rK})-\mathrm{rCl} / \mathrm{rSO}_{4}$, were calculated, Table (9). 
Table (9)Average of hydrochemical indicators for water sample.

\begin{tabular}{|c|c|c|c|c|c|c|c|c|c|c|c|c|c|c|c|c|c|c|c|c|}
\hline Item & $1 \mathrm{~A}$ & 1B & $\mathbf{2 A}$ & 2B & $\mathbf{3 A}$ & 3B & $4 \mathrm{~A}$ & 4B & $\mathbf{5 A}$ & 5B & $6 A$ & $6 \mathrm{~B}$ & $7 \mathbf{A}$ & 7B & $8 \mathrm{~A}$ & $8 \mathrm{~B}$ & 9A & 9B & $10 \mathrm{~A}$ & 10B \\
\hline $\mathrm{rK} / \mathrm{rCl}$ & 0.034 & 0.003 & 0.004 & 0.006 & 0.007 & 0.005 & 0.015 & 0.012 & 0.006 & 0.011 & 0.011 & 0.017 & 0.010 & 0.014 & 0.004 & \begin{tabular}{|l}
0.009 \\
\end{tabular} & 0.008 & 0.022 & 0.005 & 0.006 \\
\hline $\mathrm{rNa} / \mathrm{rCl}$ & \begin{tabular}{|l|}
1.13 \\
\end{tabular} & 1.04 & 1.03 & 1.16 & 1.06 & 0.87 & 1.61 & 1.42 & 1.15 & 1.18 & 0.95 & 1.17 & 1.49 & 1.49 & 1.02 & 1.39 & 1.41 & 1.53 & 1.27 & 1.15 \\
\hline $\mathrm{rCa} / \mathrm{rCl}$ & 0.23 & 0.25 & 0.17 & 0.16 & 0.16 & 0.21 & 0.16 & 0.12 & 0.23 & 0.21 & 0.13 & 0.24 & 0.09 & 0.17 & 0.40 & 0.14 & 0.20 & 0.19 & 0.24 & 0.18 \\
\hline $\mathrm{rMg} / \mathrm{rCl}$ & 0.13 & 0.03 & 0.09 & 0.05 & 0.12 & 0.12 & 0.20 & 0.08 & 0.09 & 0.09 & 0.06 & 0.11 & 0.10 & 0.16 & 0.19 & 0.07 & 0.10 & 0.10 & 0.10 & 0.06 \\
\hline $\mathrm{rCa}$ & 1.81 & 7.63 & 1 & 3.03 & 1.27 & 1.83 & 0.79 & 1.52 & 2.49 & 2.44 & 10 & 13 & 0.90 & 1.05 & 2.14 & 1.97 & 92 & 1.89 & 2.39 & 3.28 \\
\hline $\mathbf{r M g}$ & 0.55 & 0.13 & 0.55 & 0.33 & 0.79 & 0.55 & 1.27 & 0.66 & 0.40 & 0.41 & 0.48 & 0.47 & 1.11 & 0.95 & 0.47 & 0.51 & 0.52 & 0.53 & 0.42 & 0.31 \\
\hline $\mathrm{rSO}_{4} / \mathrm{rCl}$ & 0.24 & 0.16 & 0.16 & 0.07 & 0.05 & 0.09 & 0.08 & 0.06 & 0.17 & 0.12 & 0.10 & 0.21 & 0.03 & 0.06 & 0.33 & 0.07 & .16 & 0.07 & 0.11 & 0.11 \\
\hline $\mathrm{rNO}_{3} / \mathrm{rCl}$ & 0.01 & 0.001 & 0.001 & 0.01 & 0.01 & 0.001 & 0.001 & 0.01 & 0.01 & 0.01 & .01 & 01 & \begin{tabular}{|l|}
0.001 \\
\end{tabular} & 0.001 & 0.06 & 0.22 & 3 & 0.01 & 0.01 & 0.001 \\
\hline $\mathrm{rHCO}_{2} / \mathrm{rCl}$ & 0.27 & \begin{tabular}{|l|}
0.08 \\
\end{tabular} & 0.12 & 0.20 & 0.23 & \begin{tabular}{|l|}
0.10 \\
\end{tabular} & 0.76 & 0.43 & 0.29 & 0.26 & 0.41 & 0.22 & 0.68 & \begin{tabular}{|l|}
0.64 \\
\end{tabular} & 0.20 & 0.40 & 0.17 & 0.63 & 0.47 & 0.19 \\
\hline$(\mathrm{Ca}+\mathrm{Mg}) / \mathrm{HCO}_{3}$ & 1.34 & 3.51 & 2.27 & \begin{tabular}{|l|}
1.09 \\
\end{tabular} & 1.19 & 3.35 & 0.47 & 0.45 & 1.11 & 1.15 & 0.48 & 1.61 & 0.29 & 0.51 & 3.01 & 0.53 & 1.75 & 0.47 & 0.73 & 1.24 \\
\hline$(\mathrm{rNa}+\mathrm{rK}) / \mathrm{rCl}$ & 1.16 & 1.04 & 1.04 & 1.17 & 1.07 & 0.88 & 1.63 & 1.43 & 1.16 & 1.19 & 0.96 & 1.19 & 1.50 & 1.50 & 1.03 & 1.40 & 1.41 & 1.55 & 1.27 & 1.15 \\
\hline $\mathrm{Cl} / \mathrm{CO}_{3}+\mathrm{HCO}_{2}$ & 3.31 & 12.61 & & 5.09 & 3.43 & 10.13 & 1.07 & 2.14 & 3.14 & 3.85 & 2.10 & 4.59 & 1.47 & 1.57 & 12 & 2.48 & 5.75 & 1.59 & 1.98 & 5.13 \\
\hline Wate & Met. & \begin{tabular}{|l|} 
Met. \\
\end{tabular} & & & Met. & Mar. & Met. & Met. & Met. & Met. & Mar. & Met. & Met. & Met. & & $\mathbf{M}$ & & Met. & Met. & Met. \\
\hline & -0.16 & -0.04 & -0.04 & 0.17 & -0.07 & 0.13 & -0.63 & -0.43 & -0.16 & -0.19 & 0.04 & -0.19 & -0.50 & -0.50 & -0.03 & -0.40 & -0.41 & -0.55 & -0.27 & -0.15 \\
\hline CAI-2 & -0.30 & -0.16 & -0.13 & -0.62 & -0.18 & 0.68 & \begin{tabular}{|l|}
-0.62 \\
\end{tabular} & -0.80 & -0.32 & -0.48 & 0.06 & -0.43 & -0.70 & -0.72 & -0.04 & -0.57 & -1.12 & \begin{tabular}{|l|}
-0.78 \\
\end{tabular} & -0.43 & -0.50 \\
\hline
\end{tabular}

Table (9); continued

\begin{tabular}{|c|c|c|c|c|c|c|c|c|c|c|c|c|c|c|c|c|c|c|c|c|}
\hline Item & $1 \mathrm{~A}$ & 1B & $2 \mathrm{~A}$ & 2B & $3 \mathbf{A}$ & 3B & $4 A$ & 4B & $5 \mathbf{A}$ & 5B & $6 A$ & $6 B$ & $7 \mathrm{~A}$ & 7B & $8 \mathrm{~A}$ & 8B & 9A & 9B & 10A & $10 \mathrm{~B}$ \\
\hline $\mathrm{rK} / \mathrm{rCl}$ & 0.034 & 0.003 & 0.004 & 0.006 & 0.007 & 0.005 & 0.015 & 0.012 & 0.006 & 0.011 & 0.011 & 0.017 & 0.010 & 0.014 & 0.004 & 0.009 & 0.008 & 0.022 & 0.005 & 0.006 \\
\hline $\mathrm{rNa} / \mathrm{rCl}$ & 1.13 & 1.04 & 1.03 & 1.16 & 1.06 & 0.87 & 1.61 & 1.42 & 1.15 & 1.18 & 0.95 & 1.17 & 1.49 & 1.49 & 1.02 & 1.39 & 1.41 & 1.53 & 1.27 & 1.15 \\
\hline $\mathrm{rCa} / \mathrm{rCl}$ & 0.23 & 0.25 & 0.17 & 0.16 & 0.16 & 0.21 & 0.16 & 0.12 & 0.23 & 0.21 & 0.13 & 0.24 & 0.09 & 0.17 & 0.40 & 0.14 & 0.20 & 0.19 & 0.24 & 0.18 \\
\hline $\mathrm{rMg} / \mathrm{rCl}$ & 0.13 & 0.03 & 0.09 & 0.05 & 0.12 & 0.12 & 0.20 & 0.08 & 0.09 & 0.09 & 0.06 & 0.11 & 0.10 & 0.16 & 0.19 & 0.07 & 0.10 & 0.10 & 0.10 & 0.06 \\
\hline $\mathrm{rCa} / \mathrm{rMg}$ & 1.81 & 7.63 & 1.81 & 3.03 & 1.27 & 1.83 & 0.79 & 1.52 & 2.49 & 2.44 & 2.10 & 2.13 & 0.90 & 1.05 & 2.14 & 1.97 & 1.92 & 1.89 & 2.39 & 3.28 \\
\hline $\mathrm{rMg} / \mathrm{rCa}$ & 0.55 & 0.13 & 0.55 & 0.33 & 0.79 & 0.55 & 1.27 & 0.66 & 0.40 & 0.41 & 0.48 & 0.47 & 1.11 & 0.95 & 0.47 & 0.51 & 0.52 & 0.53 & 0.42 & 0.31 \\
\hline $\mathrm{rSO}_{4} / \mathrm{rCl}$ & 0.24 & 0.16 & 0.16 & 0.07 & 0.05 & 0.09 & 0.08 & 0.06 & 0.17 & 0.12 & 0.10 & 0.21 & 0.03 & 0.06 & 0.33 & 0.07 & 0.16 & 0.07 & 0.11 & 0.11 \\
\hline $\mathrm{rNO}_{3} / \mathrm{rCl}$ & 0.01 & 0.001 & 0.001 & 0.01 & 0.01 & 0.001 & 0.001 & 0.01 & 0.01 & 0.01 & 0.01 & 0.01 & 0.001 & 0.001 & 0.06 & 0.22 & 0.03 & 0.01 & 0.01 & 0.001 \\
\hline$(\mathrm{Ca}+\mathrm{Mg}) / \mathrm{HCO}$ & 1.34 & 3.51 & 2.27 & 1.09 & 1.19 & 3.35 & 0.47 & 0.45 & 1.11 & 1.15 & 0.48 & 1.61 & 0.29 & 0.51 & 3.01 & 0.53 & 1.75 & 0.47 & 0.73 & 1.24 \\
\hline$(\mathrm{rNa}+\mathrm{rK}) / \mathrm{rCl}$ & 1.16 & 1.04 & 1.04 & 1.17 & 1.07 & 0.88 & 1.63 & 1.43 & 1.16 & 1.19 & 0.96 & 1.19 & 1.50 & 1.50 & 1.03 & 1.40 & 1.41 & 1.55 & 1.27 & 1.15 \\
\hline $\mathrm{Cl} / \mathrm{CO}_{3}+\mathrm{HCO}_{3}$ & 3.31 & 12.61 & 8.54 & 5.09 & 3.43 & 10.13 & 1.07 & 2.14 & 3.14 & 3.85 & 2.10 & 4.59 & 1.47 & 1.57 & 5.12 & 2.48 & 5.75 & 1.59 & 1.98 & 5.13 \\
\hline Water Origin & Met. & Met. & Met. & Met. & Met. & Mar. & Met. & Met. & Met. & Met. & Mar. & Met. & Met. & Met. & Met. & Met. & Met. & Met. & Met. & Met. \\
\hline CAI-1 & -0.16 & -0.04 & -0.04 & -0.17 & -0.07 & 0.13 & -0.63 & -0.43 & -0.16 & -0.19 & 0.04 & -0.19 & -0.50 & -0.50 & -0.03 & -0.40 & -0.41 & -0.55 & -0.27 & -0.15 \\
\hline CAI-2 & -0.30 & -0.16 & -0.13 & -0.62 & -0.18 & 0.68 & -0.62 & -0.80 & -0.32 & -0.48 & 0.06 & -0.43 & -0.70 & -0.72 & -0.04 & -0.57 & -1.12 & -0.78 & -0.43 & -0.50 \\
\hline
\end{tabular}



Chloride ion used to know the geochemical behavior for main elements by the ratio of main elements to chloride because (Cl-) is the most dissolve ion and less influenced by physical and chemical changes in water. In addition, it is not influenced by adsorption process and exchange of ion by the clay minerals [13]. If the indicators are greater than one then the water is from meteoric origin and less than one is for water from marine origin [9]

Hydrochemical indicators' ratios, Table (9) as; $\mathrm{rNa} / \mathrm{rCl}$ range between 0.75 in well no $13 \mathrm{~B}$ and 1.76 in well no. 20A. that ratio of $81.1 \%$ of samples and raw water are $>1$ and of meteoric origin and $18.9 \%$ are $<1$ i.e. indicating halide dissolution. Also, it can be attributed to the existence of a deep recharge from the deeper aquifers in these wells, so it is of marine origin as reported [9].The $\mathrm{rK} / \mathrm{rCl}$ ratios range between 0.003 in well no.1B, and $14 \mathrm{~A}$, and 0.034 in well no.1A, also it is 0.031 in raw water sample. A ratio of $94.6 \%$ of samples are $<0.019$ that excludes addition of $\mathrm{K}$ from fertilizer application and other anthropogenic activities. And the original source of $\mathrm{Na}$ and $\mathrm{K}$ ions are the dissolution of halite $(\mathrm{NaCl})$ and sylvite $(\mathrm{KCl})$ minerals [9]. $\mathrm{rMg} / \mathrm{rCl}$ ratios range between 0.03 in well no.1B and 0.28 in well no. 20A, also it is a 0.13 in raw water sample. A ratio of $64.9 \%$ of samples and raw water are $>0.1$ and are of meteoric origin [26]. $\mathrm{rCa} / \mathrm{rCl}$ ratio range between 0.092 in well no.7A and 0.37 in well no.16A, also it is a 0.36 in raw water sample and that ratio of $100 \%$ of well samples and raw water are $>0.02$, and are of meteoric origin [26]. The values of hydrochemical coefficient $\mathrm{rSO}_{4} / \mathrm{rCl}$ range between 0.03 in well no.7A and 0.33 in well no. $8 \mathrm{~A}$, also it is a 0.16 in raw water sample and that ratio of $97.4 \%$ of well and raw water $>0.05$ are of meteoric origin. High value of this coefficient is mainly due to the dissolution processes of local terrestrial sulphate minerals present in aquifer materials [26]. $\mathrm{rNO}_{3} / \mathrm{rCl}$ ratios range between 0.001 in 14 wells and 0.22 in well no. $8 \mathrm{~B}$, also it is a 0.01 in raw water sample and that a ratio of $100 \%$ of samples and raw water are of meteoric origin as the $\mathrm{rNO}_{3} / \mathrm{rCl}$ ratio $>1.0 \times 10^{-9}[26]$. The value of the coefficient $\mathrm{rHCO}_{3} / \mathrm{rCl}$ varies between 0.03 in well no: $14 \mathrm{~A}$, to 0.98 in well no.20A, also it is a 0.25 in raw water sample. And all samples have value $<1$ which indicates predominance of $\mathrm{Cl}$ over $\mathrm{HCO}_{3}$ due to leaching process of 
chloride from its bearing minerals. The coefficient $\mathrm{rCa} / \mathrm{rMg}$ shows high values varying from 0.79 in well no.4A, to 7.63 in well no.1B, with an average of 2.06, also it is a 2.9 in raw water sample, which is more related to rainwater value 3.08 than normal sea water 0.21 , and confirms the meteoric water origin of the aquifer in the study area [11]. The coefficient $\mathrm{rMg} / \mathrm{rCa}$ shows high values varying from 0.13 in well no.1B, to 1.27 in well no.4A, with an average of 0.61 , also it is a 0.35 in raw water sample, when the $\mathrm{Mg} / \mathrm{Ca}$ ratio value $<1$ then water is of meteoric origin [27]. The value of the coefficient $(\mathrm{rCa}+\mathrm{rMg}) / \mathrm{rHCO}_{3}$ in the groundwater varies between 0.29 in well no. 7A to 10.9 in well no.14A, with an average of 1.81 in the area's groundwater. Also it is a 1.94 in raw water sample, and ratio of $83.78 \%$ of samples $>0.5$, which could be attributed to depletion of $\mathrm{HCO}_{3}$ in the aquifer systems, while a $16.2 \%<0.5$ and those around 0.5 are $32.42 \%$ and its source of $\mathrm{Ca}$ and $\mathrm{Mg}$ are mainly from the carbonate minerals [28]. The value of the hydrochemical coefficient $(\mathrm{rNa}+\mathrm{rK}) / \mathrm{rCl}$ varies between 0.76 in well no. $13 \mathrm{~B}$ to 1.76 in well no.20A, with an average of 1.196 ,also it is a 0.82 in raw water sample, and a ratio of $81.08 \%$ of samples $>1$, which indicate other sources of $\mathrm{Na}$ ions than halite and sylvite $(\mathrm{KCl})$ minerals [29]. The value of the coefficient $\mathrm{rCl} /\left(\mathrm{rHCO}_{3}+\mathrm{rCO}_{3}\right)$ in water samples have values vary between 0.92 in well No.20A and 29.14 in well. No. $14 \mathrm{~A}$, with an average value of 5.2 , also it is a 3.65 in raw water sample. The wells are categorized into four categories; slightly contaminated water $27 \%$, moderately contaminated water $54 \%$, seriously contaminated water $13.5 \%$ and highly contaminated water $5.4 \%$ [30] as shown in Table (10).

Table (10): Wells samples classification

\begin{tabular}{|c|c|c|c|c|}
\hline Category No. & I & II & III & IV \\
\hline $\mathrm{rCl} /\left(\mathrm{rHCO}_{3}+\mathrm{rCO}_{3}\right)$ & $1-2$ & $2-6$ & $6-15$ & $15-200$ \\
\hline $\begin{array}{c}\text { Category } \\
\text { description }\end{array}$ & $\begin{array}{c}\text { Slightly contaminated } \\
\text { water }\end{array}$ & $\begin{array}{c}\text { Moderately } \\
\text { contaminated } \\
\text { water }\end{array}$ & $\begin{array}{c}\text { Seriously } \\
\text { contaminated } \\
\text { water }\end{array}$ & $\begin{array}{c}\text { Highly } \\
\text { contaminated } \\
\text { water }\end{array}$ \\
\hline Wells No. & $\begin{array}{c}\mathrm{A}, 7 \mathrm{~A}, 7 \mathrm{~B}, 9 \mathrm{~B}, 10 \mathrm{~A}, \\
15 \mathrm{~B}, 17 \mathrm{~A}, 18 \mathrm{~A}, 19 \mathrm{~A} \\
\text { and } 20 \mathrm{~A}\end{array}$ & $\begin{array}{c}1 \mathrm{~A}, 2 \mathrm{~B}, 3 \mathrm{~A}, 4 \mathrm{~B}, 5 \mathrm{~A}, 5 \mathrm{~B}, \\
6 \mathrm{~A}, 6 \mathrm{~B}, 8 \mathrm{~A}, 8 \mathrm{~b}, 9 \mathrm{~A}, 10 \mathrm{~B}, \\
11 \mathrm{~A}, 11 \mathrm{~B}, 12 \mathrm{~A}, 12 \mathrm{~B}, 15 \mathrm{~A}, \\
16 \mathrm{~A}, 19 \mathrm{~B}, 20 \mathrm{~B} \text { and Raw }\end{array}$ & $\begin{array}{c}1 \mathrm{~B}, 2 \mathrm{~A}, 3 \mathrm{~B}, 13 \mathrm{~B} \text { and } \\
14 \mathrm{~B}\end{array}$ & $13 \mathrm{~A}$ and $14 \mathrm{~A}$ \\
\hline
\end{tabular}




\subsection{Index of Base Exchange}

The ion exchange between the groundwater and its host environment during residence or travel were determined using data Table (4), by calculating the chloro-alkaline indices (CAI) [14, 15].

The two chloro-alkaline indices have been calculated as shown in Table (9) Both of CAI-1and CAI-2values indicate that $18.9 \%$ water samples are positive indicates a direct exchange, whereas $81.1 \%$ samples show reverse exchange (negative indices),i.e. the $\mathrm{Ca}$ and $\mathrm{Mg}$ ions dissolute from adjacent rocks to the water reverse forward reaction[20,31].

\subsection{Groundwater Classification}

For water classifications according to Piper, Durov and Schoeller, the results in Table (4) were subjected to Aquachem v3.7 software program was used and for determining the area's groundwater type.

\section{Piper's Classification}

The results in epm were plotted on piper's diagram and the resulted diagram was compared with Langguth Classification as shown in Fig. (1).

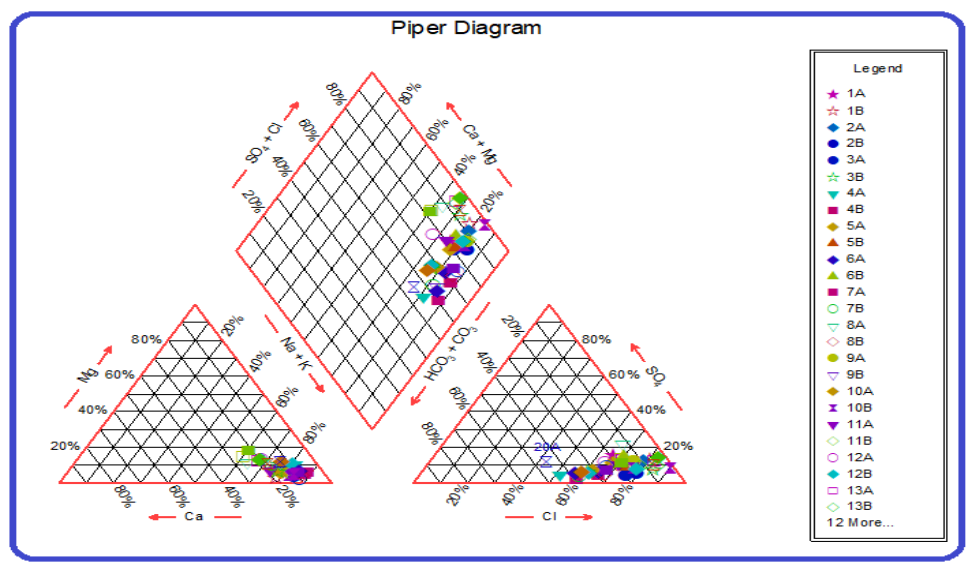

Figure (1); Piper's Classification Diagram

Piper interpretation is based on the major chemical elements of groundwater $\left(\mathrm{Ca}^{2+}, \mathrm{Mg}^{2+}, \mathrm{K}^{+}, \mathrm{Na}^{+}, \mathrm{Cl}^{-}, \mathrm{CO}_{3}{ }^{2-} \mathrm{HCO}_{3}{ }^{-}, \mathrm{SO}_{4}{ }^{2-}\right)$, to interpret general geochemical evolution pathways of groundwater in the study area. According to piper diagram, the projected onto the 
third diamond field of diagram was compared with Langguth classification revealed that all wells and raw water samples fall in field $\mathrm{G}$ and are alkaline water with prevailing of sulfate and chloride $\mathrm{Na}^{+}+\mathrm{K}^{+}-\mathrm{SO}_{4}{ }^{2-}-\mathrm{Cl}^{-}$type indicating halite weathering, as shown in Fig. (1). The dominant cation and anion of groundwater are $\mathrm{Na}$ and $\mathrm{Cl}$, respectively, the predominant salts in water samples are $(\mathrm{NaCl})$, $\left(\mathrm{Na}_{2} \mathrm{SO}_{4}\right),\left(\mathrm{MgSO}_{4}\right)$ and $\left(\mathrm{CaSO}_{4}\right)$, this is confirmed by hypothetical salt combination as shown in Table(8) [32].

\section{Durov's Classification}

The results in epm were plotted on two ternary diagrams where; the cations values were plotted perpendicularly against those of anions; the sides of the triangles form a central rectangular as shown in Fig. $(2$

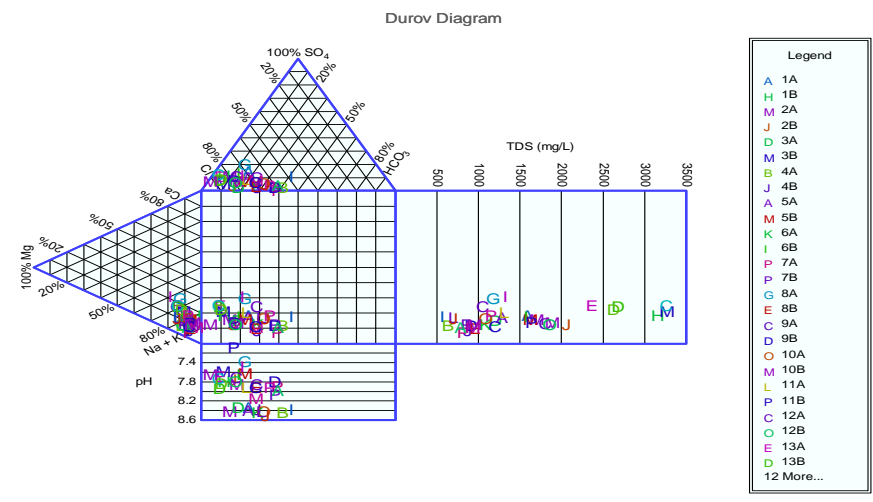

Figure (2); Durov's Classification Diagram

according to Lioyd and Heathcote division of Durov diagram all wells and raw water fall in two classes as shown in Fig. (2). Field 7; where, $\mathrm{Cl}^{-}$and $\mathrm{Na}^{+}$dominant frequently indicate end point down gradient waters through dissolution and, in Field 8; where, $\mathrm{SO}_{4}{ }^{2-}$ dominates, or anion indiscriminate and $\mathrm{Na}^{+}$dominant, is a water type not frequently encountered and indicates probable mixing or uncommon dissolution influences [16, 17].

\section{Schoeller's Classification}

Concentrations of major ions in epm, were plotted on Schoeller's diagram as shown in Fig. (3); 


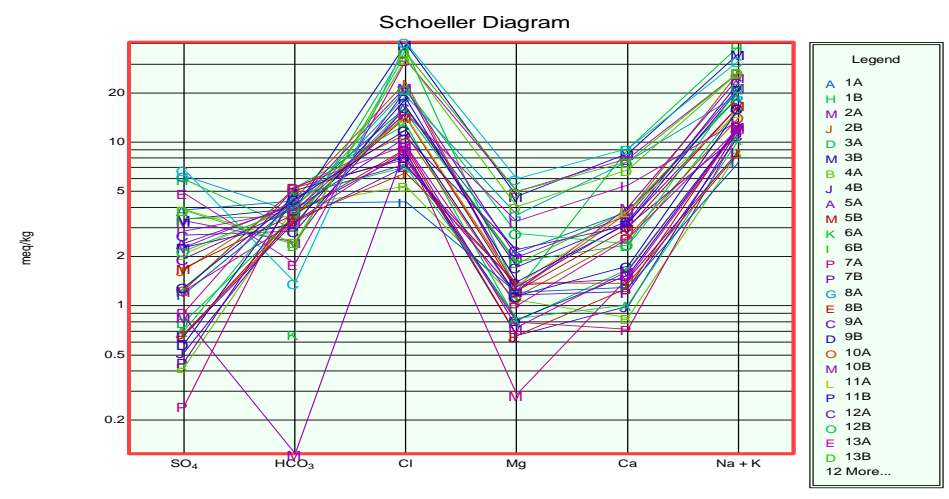

Figure (3);Schoeller's Classification Diagram

Schoeller's diagram revealed that the groundwater samples of the study area nearly had the same trend of major ion increase and decrease i.e. the similar waters exhibit similar "fingerprints" and all water samples with high $\mathrm{Na}$ content with also has high $\mathrm{Cl}$ content. The samples showed a different ionic composition that was dominated by $\mathrm{Na}^{+}$, with cationic order of abundance $\mathrm{Na}^{+}>\mathrm{Ca}^{2+}>$ $\mathrm{Mg}^{2+}$ epm, and the anionic composition was dominated by $\mathrm{Cl}^{-}>$ $\mathrm{HCO}_{3}{ }^{-}>\mathrm{SO}_{4}{ }^{2-}$ epm,i.e, the chemical composition was characterized by the $\mathrm{Na}-\mathrm{Cl}$ type as shown in Fig. (3)

\section{Gibb's Classification}

The results in Tables $(2,4)$ were subjected to Gibb's diagram and the results as shown in Table (11) Fig. (4).

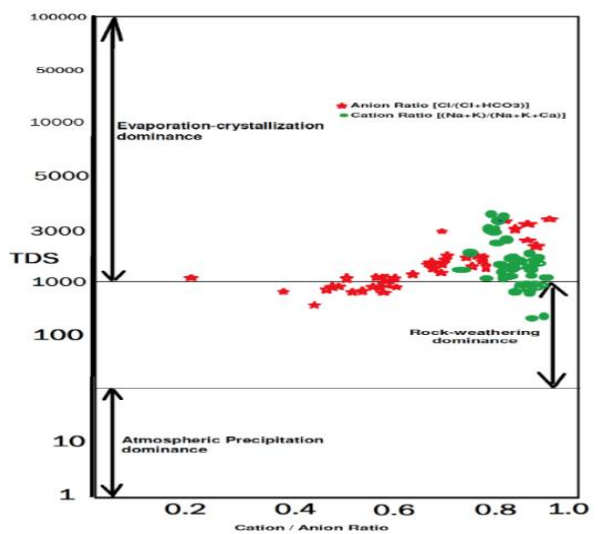

Figure (4); Gibb's

Classification diagram

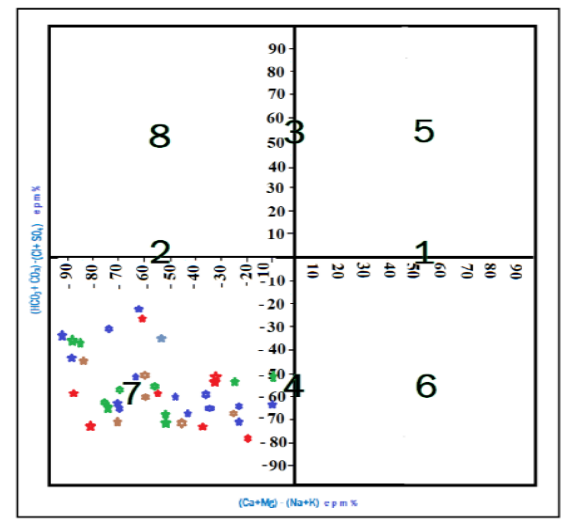

Figure (5); Chadha's diagram for study area water 



\section{Chadha's classification}

It was constructed for the results in Table (4), by plotting the difference between alkaline earth and alkali metals and the difference between weak acidic anions and strong acidic anions in epm\% as in Table (11) and Fig. (5)

Table (11); Chad ha classification's calculations

\begin{tabular}{|c|c|c|c|c|c|c|c|c|c|c|c|c|c|c|c|c|c|c|c|c|c|}
\hline tItem & $1 \mathrm{~A}$ & IB & $2 \mathrm{~A}$ & $2 \mathrm{~B}$ & $3 \mathrm{~A}$ & $3 B$ & $4 \mathrm{~A}$ & $4 B$ & $5 A$ & $5 B$ & $6 \mathrm{~A}$ & $6 \mathrm{~B}$ & $7 \mathrm{~A}$ & $7 \mathrm{~B}$ & $8 \mathrm{~A}$ & $8 B$ & $9 \mathrm{~A}$ & $9 \mathrm{~B}$ & $10 \mathrm{~A}$ & $10 \mathrm{~B}$ & $11 \mathrm{~A}$ \\
\hline$\left(\mathrm{HCO}_{3}+\mathrm{CO}_{3}\right)$ & 19.51 & 6.40 & 9.20 & 15.40 & 21.50 & 8.34 & 46.40 & 30.41 & 21.31 & 18.70 & 30.10 & 15.10 & 39.60 & 37.50 & 12.40 & 23.80 & 12.70 & 36.6 & 30.90 & 15.00 & 19.70 \\
\hline$\left(\mathrm{Cl}+\mathrm{SO}_{4}\right)$ & 80.1 & 93.3 & 90.8 & 84.1 & 77.58 & 91.68 & 53.64 & 69.09 & 78.2 & 80.8 & 69.26 & 84 & 60.26 & 62.49 & 84.1 & 63.32 & 84.88 & 62.6 & 68.19 & 84.75 & 79.3 \\
\hline$\left.\left(\mathrm{HCO}_{3}+\mathrm{CO}_{3}\right)-\mathrm{Cl}+\mathrm{SO}_{4}\right)$ & -60.59 & -86.9 & -81.6 & -68.7 & -56.1 & -83.3 & -7.24 & -38.68 & -56.9 & -62.1 & -39.16 & -68.9 & -20.66 & -24.99 & -71.7 & -39.52 & -72.18 & -26 & -37.29 & -69.75 & -59.6 \\
\hline$(\mathrm{Ca}+\mathrm{Mg})$ & 23.75 & 21.15 & 20.37 & 15.43 & 20.61 & 27.4 & 18.03 & 11.89 & 21.6 & 20.03 & 16.79 & 22.79 & 11.424 & 17.91 & 36.42 & 13.3 & 17.71 & 15.8 & 21.14 & 17.31 & 25.14 \\
\hline$(\mathrm{Na}+\mathrm{K})$ & 76.21 & 78.865 & 79.59 & 84.505 & 79.29 & 72.51 & 81.95 & 88.116 & 78.38 & 79.91 & 83.236 & 77.23 & 88.57 & 82.09 & 63.53 & 86.703 & 82.26 & 84.1 & 78.83 & 82.62 & 74.835 \\
\hline$(\mathrm{Ca}+\mathrm{Mg})-(\mathrm{Na}+\mathrm{K})$ & -52.46 & -57.7 & -59.2 & -69.07 & -58.7 & -45.1 & -63.92 & -76.26 & -56.8 & -59.88 & -66.446 & -54.44 & -77.15 & -64.18 & -27.1 & -73.4 & -64.5 & -68.2 & -57.69 & -65.32 & -49.7 \\
\hline TDS ppm & 1625 & 3187 & 1720 & 2112 & 1231 & 3283 & 663 & 925 & 1319 & 1438 & 1114 & 1651 & 846 & 891 & 1997 & 996 & 1696 & 940 & 1104 & 1910 & 1356 \\
\hline $\mathrm{Cl} / \mathrm{Cl}+\mathrm{HCO}_{\mathrm{g}}$ epm & 0.79 & 0.93 & 0.90 & 0.84 & 0.81 & 0.91 & 0.57 & 0.70 & 0.78 & 0.79 & 0.71 & 0.82 & 0.60 & 0.61 & 0.84 & 0.71 & 0.85 & 0.61 & 0.68 & 0.84 & 0.78 \\
\hline $\mathrm{Na}+\mathrm{K} / \mathrm{Na}+\mathrm{K}+\mathrm{Ca}$ epm & 0.83 & 0.81 & 0.86 & 0.88 & 0.87 & 0.80 & 0.91 & 0.92 & 0.84 & 0.85 & 0.88 & 0.83 & 0.94 & 0.90 & 0.72 & 0.91 & 0.88 & 0.89 & 0.84 & 0.86 & 0.80 \\
\hline
\end{tabular}

]

\section{Table (11): continued}

\begin{tabular}{|c|c|c|c|c|c|c|c|c|c|c|c|c|c|c|c|c|c|}
\hline Item & 11B & $12 \mathrm{~A}$ & $12 \mathrm{~B}$ & $13 \mathrm{~A}$ & $13 \mathrm{~B}$ & $14 \mathrm{~A}$ & $14 \mathrm{~B}$ & $15 \mathrm{~A}$ & 15B & $16 \mathrm{~A}$ & $17 \mathrm{~A}$ & $18 \mathrm{~A}$ & $19 \mathrm{~A}$ & 19B & $20 \mathrm{~A}$ & $20 \mathrm{~B}$ & Raw \\
\hline$\left(\mathrm{HCO}_{8}+\mathrm{CO}_{3}\right)$ & 13.20 & 23.50 & 14.50 & 4.88 & 5.91 & 2.80 & 6.56 & 23.60 & 33.60 & 17.50 & 38.10 & 34.34 & 37.06 & 25.30 & 45.90 & 26.80 & 19.05 \\
\hline$\left(\mathrm{Cl}+\mathrm{SO}_{4}\right)$ & 6.48 & 75.8 & 85.2 & 92.6 & 92.07 & 95.2 & 92.2 & 74 & 66.3 & 81.3 & 61.89 & 65.66 & 62.85 & 74.7 & 54 & 73.1 & 80.5 \\
\hline$\left.\left(\mathrm{HCO}_{3}+\mathrm{CO}_{3}\right)-\mathrm{Cl}+\mathrm{SO}_{4}\right)$ & -73.28 & -52.3 & -70.7 & -87.72 & -86.16 & -92.4 & -85.64 & -50.4 & -32.7 & -63.8 & -23.79 & -31.32 & -25.79 & -49.4 & -8.1 & -46.3 & -61.45 \\
\hline$(\mathrm{Ca}+\mathrm{Mg})$ & 17.8 & 32.47 & 19.21 & 32.9 & 32.1 & 32.5 & 28.7 & 12.66 & 22.59 & 39.2 & 15.1 & 23.8 & 24.01 & 15.2 & 24.7 & 15.81 & 37.27 \\
\hline$(\mathrm{Na}+\mathrm{K})$ & 2.19 & 67.5 & 80.75 & 67.1 & 67.78 & 67.48 & 71.3 & 87.31 & 77.36 & 60.7 & 84.9 & 76.13 & 75.96 & 84.82 & 75.2 & 84.13 & 62.67 \\
\hline$(\mathrm{Ca}+\mathrm{Mg})-(\mathrm{Na}+\mathrm{K})$ & -64.39 & -35.06 & -61.54 & -34.2 & -35.68 & -34.98 & -42.6 & -74.65 & -54.77 & -21.55 & -69.8 & -52.33 & -51.95 & -69.62 & -50.5 & -68.32 & -25.4 \\
\hline TDS ppm & 1669 & 1081 & 1881 & 2400 & 2707 & 3280 & 2652 & 943 & 1182 & 1404 & 821 & 740 & 749 & 1224 & 643 & 966 & 2858 \\
\hline $\mathrm{Cl} / \mathrm{Cl}+\mathrm{HCO}_{3}$ epm & 0.85 & 0.73 & 0.84 & 0.95 & 0.94 & 0.97 & 0.93 & 0.73 & 0.64 & 0.80 & 0.60 & 0.68 & 0.66 & 0.73 & 0.50 & 0.71 & 0.80 \\
\hline $\mathrm{Na}+\mathrm{K} / \mathrm{Na}+\mathrm{K}+\mathrm{Ca}$ epm & 0.87 & 0.78 & 0.90 & 0.77 & 0.78 & 0.77 & 0.80 & 0.89 & 0.83 & 0.71 & 0.91 & 0.86 & 0.86 & 0.90 & 0.85 & 0.88 & 0.69 \\
\hline
\end{tabular}



Gibb's diagram showed that the distribution of sample points residing in the central part of the plot based on ratios of $(\mathrm{Na}+\mathrm{K}) /(\mathrm{Na}+\mathrm{K}+\mathrm{Ca})$ and $\mathrm{Cl} /\left(\mathrm{Cl}+\mathrm{HCO}_{3}\right)$ as a function of TDS, reflected the supremacy of weathering of rocks with influence of evaporation crystallization in controlling geochemistry of water samples from the study area (Fig.4)[4]. None of samples lie in the lower side of the boomerang, where water composition is dominated by atmospheric precipitation process.

Chadha's diagram showed that most of samples fall in seventh subfield of the diagram which reveals that alkali metals $(\mathrm{Na}, \mathrm{K})$ and strong acidic anions $\left(\mathrm{Cl}, \mathrm{SO}_{4}\right)$ exceed over alkaline earths $(\mathrm{Ca}, \mathrm{Mg})$ and weak acidic anions $\left(\mathrm{HCO}_{3}\right)$, respectively Fig. (5). The positions of data points in the diagram represent $\mathrm{Na}-\mathrm{Cl}^{-}-\mathrm{SO}_{4}{ }^{2-}$ type. The water of this type has high TDS value due to dissolution of soluble evaporated minerals such as halite and gypsum, and $\mathrm{Na}-\mathrm{Ca}$ exchange reactions occurring between the groundwater and fined grain-sediments.

\subsection{Hydrochemical Faces in the Study Area}

Hydrochemical faces are a function of solution kinetics, rock-water interactions, geology and contamination sources, and are identified from Kurolov, Piper, Durov and Chadha diagrams and chebotarev [21, 33, 34, 35]. The Kurolov hydrochemical formula reveals that there are six hydrochemical formulas and the main water face is $\mathrm{Na}-\mathrm{Cl}$. But according a more distinct ways as; Chebotarev classification depending on TDS content, Table (2), the water type is ranges between $\mathrm{HCO}_{3}-\mathrm{Cl}$,fairly fresh water(F3), to $\mathrm{Cl}-\mathrm{SO}_{4}$ face,slightly brackish water(B1).According Piper area's waters are alkaline water with prevailing of sulfate and chloride $\mathrm{Na}+\mathrm{K}-\mathrm{SO}_{4}+\mathrm{Cl}$ face, also, according Durov $\mathrm{Cl}-\mathrm{SO}_{4}-\mathrm{Na}$ are dominant face, but according to Chadha $\mathrm{Na}-\mathrm{Cl}-\mathrm{SO}_{4}$ type is the prevailing face.

\section{Conclusion}

The hydrochemical class and water type of groundwater in West Assiut Electric Power Plant's area(with respect to the accurate and valid physiochemical and chemically related properties) are determined using the hydrochemical formulae's (Kurolov formula, hypothetical salts), hydrochemical indices and water type 
identification programs as Piper, Durov, Schoeller, Gibb, and Chadha were applied for deciding the water class and type. Kurolov formula showed six groups (assemblages) of salt combination, while combination between major anions and cations reveals the formation of seven assemblages of hypothetical salts combinations. Hydrochemical indicators' ratios revealed that the recharge from Nile River is very weak and may be a deep recharge from the deeper aquifers in these wells. The chloro-alkaline indices indicate that the major reaction is the dissolution of $\mathrm{Ca}$ and $\mathrm{Mg}$ to groundwater (negative indices) and the precipitation of sodium and potassium into the soil is the minor reaction (positive indices). According to Piper, area's groundwater are alkaline water with prevailing of sulfate and chloride $\mathrm{Na}+\mathrm{K}-\mathrm{SO}_{4}+\mathrm{Cl}$ face, also, according to Durov $\mathrm{Cl}+\mathrm{SO}_{4}-\mathrm{Na}$ are dominant face, also according to Chadha $\mathrm{Na}-$ $\mathrm{Cl}+\mathrm{SO}_{4}$ type is the prevailing face. While Schoeller classified it to $\mathrm{Na}$ and $\mathrm{Cl}$ and the families are $\mathrm{Na}-\mathrm{Cl}$ and $\mathrm{Gibb}$ reflected the supremacy of weathering of rocks with influence of evaporation crystallization in controlling geochemistry of groundwater in the study area.

The finding of the above work is useful for Determination of pollutants in the groundwater wells of the plant, and consequently the method of water treatment, which is chosen according to the type of use: for agriculture, irrigation, industrial and building Purposes, human drinking and livestock Purposes.

The research is useful in guiding orgiving an idea for those who want to use groundwater in the Western Desert region and the borders of the Assiut Governorate region on water components and how to treat .

\section{References}

1.Khan, Stuart J., Murchland, David, Rhodes, Michelle and Waite, T. David, (2009), "Management of Concentrated Waste Streams from High-Pressure Membrane Water Treatment Systems", Critical Reviews in Environmental Science and Technology 39:5, 367 - 415.

2.Todd, D.K. (1980) Groundwater hydrology. 2nd ed,Wiley, New York.

3.Winter, Th. C., Harvey, J. W., Lehn Franke, O. and Alley, W. M., (1998) "Ground Water and Surface water A Single Resource, U.S.G.S. Circular 1139: 79. 
4.Ravikumar, P., Somashekar, R.K and Prakash, K.L. (2015) "A comparative study on usage of Durov and Piper diagrams to interpret hydrochemical processes in groundwater from SRLIS river basin," Karntaka, India Available online at (Elixir International Journal) Earth Science Elixir Earth Sci. 80, 31073-31077.

5.APHA ( 2017). (American Public Health Association), American water works association (AWWA) and Water Environmental Federation (WEF). Standard methods for the examination of water and wastewater, $23^{\text {nd }}$ Edition.

6.APHA (1998). Standard Methods of the Examination of Water and Wastewater. 20th ed., Washington D.C., American Public Health Association.

7.APHA (2005) (American Public Health association)...Methods for the examination of water and wastewater, $21^{\text {st }}$ edition.

8.Lind, O. T. ( 1979). Handbook at Common Methods in Limnology C.V. Mosby St. Louis.

9.Ivanov, V.V., Barbanov, L. N., and Plotnikova, G. N. (1968). The main genetic types of the earths crust mineral water and their distribution in the USSR. Inter. Geol. Cong. of 23rd. Sessions Czechoslovakia, V. 12.p33.

10. Mohamed Yousif, Olaf Bubenzer, (2012)"Perched groundwater at the northwestern coast of Egypt": a case study of the Fuka Basin. Appl Water Sci 2:15-28.

11. Mohamed kamel Fattah (2017) "Evaluation of Water Resources in Wadi El Natrun, Western Desert, Egypt”. International Journal of Environment, Agriculture and Biotechnology (IJEAB) Vol-2, Issue1, Jan-Feb.

12. Collins, A. G. (1975) "Ge chemistry of oil- field water." Development in Petroleum science, No.11, Elsevier, Amsterdam, Holland, , 496p.

13. Levy, D. B and Kearney W. F. (1974) Irrigation of native rangeland using treated waste water from in situ uranium processing J. Environ. Quality. 28, 208-217.

14. Schoeller H. (1967) Geochemistry of G. water. An international guide for research and practice, UNESCO, Chap.15,p1-

15. Hedge, G. V., (2006) Evaluation of chemical quality of groundwater resource in Dharward districts Karnataka. Journal of Geological Society of India 67, p. 4-58. 
16. R. A. Akoachere, Y. M. Ngwese, S. E. Egbe, T. A. Eyong, S. N. Edimo, D. B. Tambe, (2018) "Groundwater Monitoring in the Gneisso-Basaltic Fractured Rock Aqu ferous Formations of Kumba, Southwest Region Cameroon: Seasonal Variations in the Aqueous Geochemistry and Water Quality" Journal of Geoscience and Environment Protection, 6, 18-50.

17. Lloyd, J. A., \& Heathcote, J. A., (1985) "Natural Inorganic Hydrochemistry in Relation to Groundwater: An Introduction", New York: Oxford University Press, (p. 296).

18. Schoeller R.: Les eaux Souterraines, Msson and Cie, Paris (1962), 642pp.

19. Gibb, R.,1971. Mechanism controlling world river water chemistry: vapor. - crystallize. process. Science 172, 871-872.

20. Chadha DKA, (1999) "Proposed new diagram for geochemical classification of natural waters and interpretation of chemical data." Hydrogeol J, 7(5):431-439.

21. Chebotarev, I.I., (1955), "Metamorphism of natural water in the crust of weathering." Geochim. Cosmochim. Acta, v.8, pp.22-48, 137- 170, 198-212.

22. Roger B. Baird, Andrew D. Eaton, Eugene W. Rice (2017), "standard methods for the examination of water wastewater", 23rd Edition

23. Hem, J.D., (1985) "Study and interpretation of the chemical characteristics of natural water," 3rd. ed. U.S.G.S. water supply paper., 2254. 263p.

24. Al-Hamadani, J.A.,( 2009). Hydrochemical effect of ground water due to irrigation and drainage projects in Tawuq sub-Basin (South of Karkuk North of Iraq). Unpublished M.Sc. University of Baghdad, College of Science. 120pp.

25. Valerie B.Garcia, Katharine D., Katherine G., Andrew T. "Treating Brackish Groundwater in Texas: A Comparison of Reverse Osmosis and Nanofiltration" Final Report Submitted to the Texas Water Development Board, U.S. Department of the Interior Bureau of Reclamation, May 2015.

26. Mahmoud K., Corinne Le Gal La Salle, Joel L., ASTER team, Amad M., Patrick V., Aurelie N., Roland S., (2013) "Origin of groundwater salinity (current seawater vs. saline deep water) in a coastal karst aquifer based on $\mathrm{Sr}$ and $\mathrm{Cl}$ isotopes. Case study of the La Clape massif (southern France)" Applied Geochemistry 37-212227 
27. Hem, J. D., (1992). Study and Interpretation of the Chemical Characteristics of Natural Water. U.S. Geological Survey WaterSupply Paper 2254.

28. Rajmohan $\mathbf{N}$ and Elango L. (2004) "Identification and evolution of hydrogeochemical processes in the groundwater environment in an area of the Palar and Cheyyar River Basins, Southern India". Environ Geology, ,46:47-61

29. Hesham A. Ezzeldin, Ahmad Kamal, Aya M. Reda, Khairia Guindy and Ibrahim El-Shamy, (2018). "Assessment of Factors Affecting the Groundwater Quality in El-Hammam Area, North-West Coast of Egypt" Middle East Journal of Applied Sciences, Volume: 08- p 798-819 | Issue :03 |July-Sept.|

30. Mohamed el Alfy, Broder Merkel, (2006) "Hydrochemical Relationships and Geochemical Modeling Of Ground Water In Al Arish Area, North Sinai, Egypt', Journal of American Institute of Hydrology, Hydrological Science and Technology, 22(1-4) 47-62.

31. Freeze, R.A. and Cherry, G.E. (1979). "Groundwater. Prentice Hall, Englewood Cliffs", New Jersey, U.S.A, 460.

32. Alassane, A., Trabelsi, R., Dovonon, L.F., Odeloui, D.J., Boukari, M., Zouari, K. and Mama, D. (2015) Chemical Evolution of the Continental Terminal Shallow Aquifer in the South of Coastal Sedimentary Basin of Benin (West- Africa) Using Multivariate Factor Analysis. Journal of Water Resource and Protection, 7, 496515.

33. Karanth K. R. (2008) "G.water Assessment Development and Management" Tata McGraw-Hill Offices, New Delhi,p 720.

34. K. Srinivasamoorthy, M. Gopinath, S. Chidambaram, M. Vasanthavigar, V.S. Sarma, (2014) "Hydrochemical characterization and quality appraisal of groundwater from Pungar sub basin, Tamilnadu, India, Journal of King Saud University Science 26, 37-52.

35. Stevanovic Z. \& Iurkiewicz A. (2009) "G. water management in northern Iraq."Hydrogeology Journal, 17:367-378. 


\section{الالإمس العزبى}

التصنيف الهيدروكيميائي للامياه الجوفية في هنطقة همطة كهرباء غرب

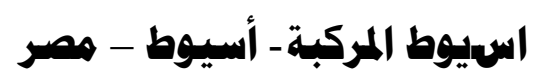

عبدالعال محمد جابر 1- عادل محمد كمال الدين2 - حسانين مناع حساتين3 - رامي محمد دسوقي4

$$
\begin{aligned}
& \text { 1 كالية تكنولوبيا صناعة السكر والصنا عاتـ التكاملية } \\
& 2 \text { قسم:الكيمياء-بكلية العلوم بـامعة أسيوط } \\
& 3 \text { قسمالكبيمياء- مهطة كمرباء غرب اسبيوطالمركبة }
\end{aligned}
$$

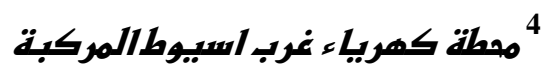

المباه الجوفية هي المصدر الرئيسي للمياه المستخدمة في محطة كهرباء غرب

اسبوط المركبة، لذلك سوف نقوم بدراسة التصنيف الهيدروكيميائي ونوعية المياه الجوفية

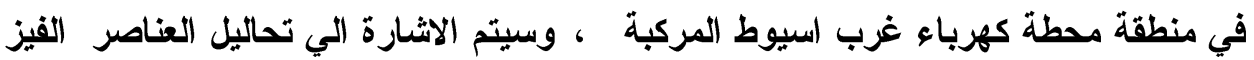

$$
\text { وكيميائية و الكيميائية والخصائص الكيميائية }
$$

وكي نسـتطيع ان نقرر تصنيف المياه الجوفية سنقوم بتطبيق الصيغ الهيدروكيميائية مثل وكئل صيغة كروبوف ( Kurolov formula ) والاملاح الافتر اضية (hypothetical salts) و

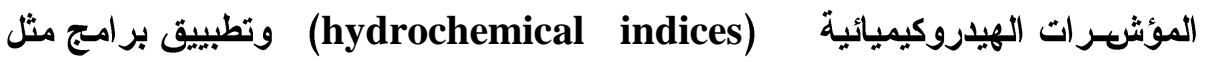

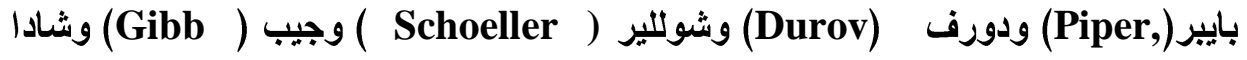
لتحديد نوع المياه. وتمكن البحث من تحديد الملوثات داخل مياه الابار الجوفية (Chadha)

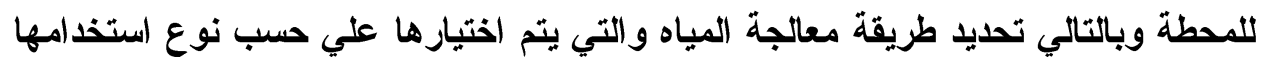
، ويفيد البحث في توجيه أو إعطاء فطرة لمن يريد استخدام المياه الجوفية في منطقة (الصحر اء الغربية وحدود منطقة محافظة اسيوط علي مكونات المياه وكيفية معالجتها)

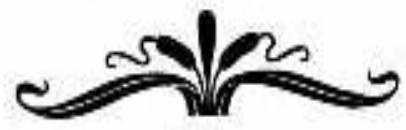

